\title{
Field Correlators in Abelian-Projected Theories and Stochastic Vacuum Model
}

\author{
Dmitri Antonov * $\dagger$ \\ INFN-Sezione di Pisa, Universitá degli studi di Pisa, \\ Dipartimento di Fisica, Via Buonarroti, 2 - Ed. B - 56127 Pisa, Italy
}

\begin{abstract}
The bilocal electric field strength correlators in Abelian-projected $S U(2)$ - and $S U(3)$-theories are derived with accounting for the contributions to these quantities brought about by the virtual vortex loops, built out of the dual Nielsen-Olesen strings. Owing to the screening of magnetic charge of the dual vector bosons in the gas of such loops, these bosons become heavier, which leads to the respective change of the correlation length of the vacuum in the models under study. Besides that, it turns out that such a screening leads also to the appearance of the long-range contribution to one of the two coefficient functions, which parametrize the bilocal correlator within the Stochastic Vacuum Model. Finally, the modifications of the propagators of the dual vector bosons, inspired by the correlation effects in the gas of vortex loops, are also discussed.
\end{abstract}

Keywords: QCD, Confinement, Phenomenological Models, Nonperturbative Effects

${ }^{*}$ Permanent address: Institute of Theoretical and Experimental Physics, B. Cheremushkinskaya 25, RU-117 218 Moscow, Russia.

†Tel.: + 39050844 536; Fax: + 39050844 538; E-mail address: antonov@difi.unipi.it 


\section{Introduction}

Stochastic Vacuum Model (SVM) [1] is nowadays commonly recognized to be one of the most promising nonperturbative approaches to QCD (see Ref. [2] for recent reviews). Within the so-called bilocal or Gaussian approximation, well confirmed by the existing lattice data [3, 4], this model is fully described by the irreducible bilocal gauge-invariant field strength correlator (cumulant), $\left\langle\left\langle F_{\mu \nu}(x) \Phi\left(x, x^{\prime}\right) F_{\lambda \rho}\left(x^{\prime}\right) \Phi\left(x^{\prime}, x\right)\right\rangle\right\rangle$. Here, $F_{\mu \nu}=\partial_{\mu} A_{\nu}-\partial_{\nu} A_{\mu}-i g\left[A_{\mu}, A_{\nu}\right]$ stands for the Yang-Mills field strength tensor, $\Phi(x, y) \equiv \frac{1}{N_{c}} \mathcal{P} \exp \left(i g \int_{y}^{x} A_{\mu}(u) d u_{\mu}\right)$ is a parallel transporter factor along the straight-line path, and $\left\langle\left\langle\mathcal{O O}^{\prime}\right\rangle\right\rangle \equiv\left\langle\mathcal{O O}^{\prime}\right\rangle-\langle\mathcal{O}\rangle\left\langle\mathcal{O}^{\prime}\right\rangle$ with the average defined w.r.t. the Euclidean Yang-Mills action. It is further convenient to parametrize the bilocal cumulant by the two coefficient functions [1], 2] as follows:

$$
\begin{gathered}
\frac{g^{2}}{2}\left\langle\left\langle F_{\mu \nu}(x) \Phi\left(x, x^{\prime}\right) F_{\lambda \rho}\left(x^{\prime}\right) \Phi\left(x^{\prime}, x\right)\right\rangle\right\rangle=\hat{1}_{N_{c} \times N_{c}}\left\{\left(\delta_{\mu \lambda} \delta_{\nu \rho}-\delta_{\mu \rho} \delta_{\nu \lambda}\right) D\left(\left(x-x^{\prime}\right)^{2}\right)+\right. \\
\left.+\frac{1}{2}\left[\partial_{\mu}^{x}\left(\left(x-x^{\prime}\right)_{\lambda} \delta_{\nu \rho}-\left(x-x^{\prime}\right)_{\rho} \delta_{\nu \lambda}\right)+\partial_{\nu}^{x}\left(\left(x-x^{\prime}\right)_{\rho} \delta_{\mu \lambda}-\left(x-x^{\prime}\right)_{\lambda} \delta_{\mu \rho}\right)\right] D_{1}\left(\left(x-x^{\prime}\right)^{2}\right)\right\} .
\end{gathered}
$$

After that, setting for the nonperturbative parts of the $D$ - and $D_{1}$-function various Ansätze, one can employ SVM for precise calculations of the processes of high-energy scattering [5] or test these Ansätze in the lattice experiments [3, 4. However from the pure field-theoretical point of view, it remains a great challenge to derive the coefficient functions analytically. Unfortunately, in this way no big progress has up to now been achieved in the QCD itself. There have rather been derived some relations between cumulants of various orders [6], which might be useful only in testing the IR asymptotic behaviours of the coefficient functions.

Contrary to that, more progress has recently been achieved in a derivation of the bilocal cumulant in some models allowing for an analytical description of confinement. Those include Abelian-projected (AP) $S U(2)$ - [7] and $S U(3)$ [8] theories, as well as compact QED [9]. The bilocal field strength cumulant in these theories has been studied in Refs. [10, 11, 12], respectively (see Ref. [13] for a review). The present paper also follows this line of investigations and is devoted to the improvement of calculations of the bilocal cumulant in AP theories. This improvement is based on the well known fact [14] that in the case of zero temperature under study, Abrikosov vortices [15] in the Ginzburg-Landau theory (dual Nielsen-Olesen strings [16] in our 4D-case) form bound states, built out of a vortex and an antivortex, which are usually referred to as vortex dipoles (vortex loops in 4D). Such vortex loops are short living (virtual) objects, whose typical sizes are much smaller than the typical distances between them. This means that similarly to monopoles in 3D compact QED, vortex loops form a dilute gas. The summation over the grand canonical ensemble of vortex loops in such a dilute gas approximation was performed in Ref. [17] for the case of the usual Abelian Higgs model (dual AP $S U(2)$-gluodynamics) in 3D and 4D and then extended to the case of the 4D AP $S U(3)$-gluodynamics in Ref. [18 I. On the other hand, in all the investigations of the bilocal field strength cumulants in AP theories, performed in Refs. [10, 11], the contribution of vortex loops to the partition function, and consequently to the cumulants themselves, was disregarded. As it will be demonstrated in the next Section, this

\footnotetext{
${ }^{1}$ Note that in the case of the 2D Abelian Higgs model, such a summation has for the first time been performed in Ref. [19].
} 
approximation is really valid, since it is equivalent to another one, which states that the typical sizes of vortex loops are negligibly small. However, such a neglection of the contribution of vortex loops makes the calculations of the field strength correlators, performed in the above mentioned papers, essentially classical. The improvement of these calculations, presented in this paper, is just based on accounting for the correlations in the gas of vortex loops. Clearly, such correlations are of the quantum origin, as well as the grand canonical ensemble of virtual vortex loops itself. Besides that, we shall also evaluate the contributions of vortex loops to the propagators of the dual vector bosons and discuss the so-emerging modifications of the respective classical expressions.

The paper is organized as follows. In the next Section, we shall firstly review the main aspects of a derivation of AP theories, necessary for the future purposes. Secondly, we shall review the main results of a calculation of electric field strength correlators in the approximation when the contribution of vortex loops to these quantities is disregarded. In Section 3, after a brief review of the properties of the grand canonical ensemble of vortex loops, we shall evaluate the contribution of these objects to the field strength correlators. In Section 4, the same will be done for the propagators of the dual vector bosons. The main results of the paper are summarized in Conclusion. In the Appendix, some technical details of the calculation of a certain typical integral from the main text are outlined.

\section{Electric Field Strength Correlators in the Absence of Vortex Loops Revisited}

\section{$2.1 \quad$ The Models}

To derive from the gluodynamics Lagrangian the IR effective $S U(2)$ - and $S U(3)$ AP theories, based on the assumption of condensation of Cooper pairs of AP monopoles, one usually employs the so-called Abelian dominance hypothesis [20]. It states that the off-diagonal (in the sense of the Cartan decomposition) fields can be disregarded, since after the Abelian projection those can be shown to become very massive and therefore irrelevant to the IR region, where confinement holds. Let us start our analysis with the $S U(2)$-theory. Then, the action describing the rest, diagonal, fields and AP monopoles reads

$$
S_{\text {eff. }}\left[a_{\mu}, f_{\mu \nu}^{\mathrm{m}}\right]=\frac{1}{4} \int d^{4} x\left(f_{\mu \nu}+f_{\mu \nu}^{\mathrm{m}}\right)^{2} \text {. }
$$

Here, $a_{\mu} \equiv A_{\mu}^{3}, f_{\mu \nu}=\partial_{\mu} a_{\nu}-\partial_{\nu} a_{\mu}$, and the monopole field strength tensor $f_{\mu \nu}^{\mathrm{m}}$ obeys Bianchi identities modified by monopoles, $\partial_{\mu} \tilde{f}_{\mu \nu}^{\mathrm{m}} \equiv \frac{1}{2} \varepsilon_{\mu \nu \lambda \rho} \partial_{\mu} f_{\lambda \rho}^{\mathrm{m}}=j_{\nu}^{\mathrm{m}}$. The monopole currents $j_{\mu}^{\mathrm{m}}$ 's should eventually be averaged over in the sense, which will be specified below.

To proceed with the investigation of the monopole ensemble, it is reasonable to cast the theory under study to the dual form. This yields the following expression for the partition function:

$$
\mathcal{Z}=\left\langle\int \mathcal{D} B_{\mu} \exp \left[-\int d^{4} x\left(\frac{1}{4} F_{\mu \nu}^{2}-i B_{\mu} j_{\mu}^{\mathrm{m}}\right)\right]\right\rangle_{j_{\mu}^{\mathrm{m}}}
$$

where $B_{\mu}$ is the magnetic vector-potential dual to the electric one, $a_{\mu}$, and $F_{\mu \nu}=\partial_{\mu} B_{\nu}-\partial_{\nu} B_{\mu}$. Once the $j_{\mu}^{\mathrm{m}}$-dependence of the action became explicit, it is now possible to set up the properties of the monopole ensemble. To describe the condensation of monopole Cooper pairs, it is first necessary to specify $j_{\mu}^{\mathrm{m}}$ as the collective current of $N$ of those: 


$$
j_{\mu}^{\mathrm{m}(N)}(x)=2 g_{m} \sum_{n=1}^{N} \oint d x_{\mu}^{n}(s) \delta\left(x-x^{n}(s)\right) .
$$

Here, the world line of the $n$-th Cooper pair is parametrized by the vector $x_{\mu}^{n}(s)$, and $g_{m}$ is the magnetic coupling constant, related to the QCD coupling constant $g$ via the topological quantization condition $g g_{m}=4 \pi n$ with $n$ being an integer. In what follows, we shall for concreteness restrict ourselves to the monopoles possessing the minimal charge, i.e. set $n=1$, although the generalization to an arbitrary $n$ is straightforward. Secondly, it is necessary to set for the measure $\langle\ldots\rangle_{j_{\mu}^{\mathrm{m}}}$ the following expression [21]:

$$
\begin{gathered}
\left\langle\exp \left(i \int d^{4} x B_{\mu} j_{\mu}^{\mathrm{m}}\right)\right\rangle_{j_{\mu}^{\mathrm{m}}}=1+\sum_{N=1}^{\infty} \frac{1}{N !}\left[\prod_{n=1}^{N} \int_{0}^{+\infty} \frac{d s_{n}}{s_{n}} \mathrm{e}^{4 \lambda \eta^{2} s_{n}} \int_{u(0)=u\left(s_{n}\right)} \mathcal{D} u\left(s_{n}^{\prime}\right)\right] \times \\
\times \exp \left\{\sum_{l=1}^{N} \int_{0}^{s_{l}} d s_{l}^{\prime}\left[-\frac{1}{4} \dot{u}^{2}\left(s_{l}^{\prime}\right)+2 i g_{m} \dot{u}_{\mu}\left(s_{l}^{\prime}\right) B_{\mu}\left(u\left(s_{l}^{\prime}\right)\right)\right]-4 \lambda \sum_{l, k=1}^{N} \int_{0}^{s_{l}} d s_{l}^{\prime} \int_{0}^{s_{k}} d s_{k}^{\prime \prime} \delta\left[u\left(s_{l}^{\prime}\right)-u\left(s_{k}^{\prime \prime}\right)\right]\right\} .
\end{gathered}
$$

Here, the vector $u_{\mu}\left(s_{n}^{\prime}\right)$ parametrizes the same contour as the vector $x_{\mu}^{n}(s)$. Clearly, the world-line action standing in the exponent on the R.H.S. of Eq. (田) contains besides the usual free part also the term responsible for the short-range repulsion of the trajectories of Cooper pairs. Equation (田) can further be rewritten as an integral over the dual Higgs field, describing magnetic Cooper pairs, as follows:

$$
\left\langle\exp \left(i \int d^{4} x B_{\mu} j_{\mu}^{\mathrm{m}}\right)\right\rangle_{j_{\mu}^{\mathrm{m}}}=\int \mathcal{D} \Phi \mathcal{D} \Phi^{*} \exp \left\{-\int d^{4} x\left[\frac{1}{2}\left|D_{\mu} \Phi\right|^{2}+\lambda\left(|\Phi|^{2}-\eta^{2}\right)^{2}\right]\right\},
$$

where $D_{\mu}=\partial_{\mu}-2 i g_{m} B_{\mu}$ is the covariant derivative 2. Finally, substituting Eq. (5) into Eq. (3), we arrive at the following IR effective AP theory of the $S U(2)$-gluodynamics:

$$
\mathcal{Z}=\int|\Phi| \mathcal{D}|\Phi| \mathcal{D} \theta \mathcal{D} B_{\mu} \exp \left\{-\int d^{4} x\left[\frac{1}{4} F_{\mu \nu}+\frac{1}{2}\left|D_{\mu} \Phi\right|^{2}+\lambda\left(|\Phi|^{2}-\eta^{2}\right)^{2}\right]\right\}
$$

where $\Phi(x)=|\Phi(x)| \mathrm{e}^{i \theta(x)}$. Clearly, as soon as we have disregarded the off-diagonal degrees of freedom and demanded the condensation of monopole Cooper pairs, this theory is nothing else, but just the dual Abelian Higgs model.

Analogous considerations can be applied to the $S U(3)$-gluodynamics. The only difference is that since the $S U(3)$-group has two diagonal generators, the resulting AP theory will also be $[U(1)]^{2}$ magnetically gauge-invariant. Within the Abelian dominance hypothesis, the initial action reads

$$
S_{\text {eff. }}\left[\mathbf{a}_{\mu}, \mathbf{f}_{\mu \nu}^{\mathrm{m}}\right]=\frac{1}{4} \int d^{4} x\left(\mathbf{f}_{\mu \nu}+\mathbf{f}_{\mu \nu}^{\mathrm{m}}\right)^{2}
$$

and after the dualization we have for the partition function (cf. Eq. (3)):

\footnotetext{
${ }^{2}$ A seeming divergency at large proper times produced in Eq. (1) by the factor $\mathrm{e}^{4 \lambda \eta^{2} s_{n}}$ is actually apparent, since the last term in the exponent on the R.H.S. of this equation yields the desired damping.
} 


$$
\mathcal{Z}=\left\langle\int \mathcal{D} \mathbf{B}_{\mu} \exp \left\{-\int d^{4} x\left[\frac{1}{4} \mathbf{F}_{\mu \nu}^{2}-i \mathbf{B}_{\mu} \mathbf{j}_{\mu}^{\mathrm{m}}\right]\right\}\right\rangle_{\mathbf{j}_{\mu}^{\mathrm{m}}} .
$$

Here, $\mathbf{F}_{\mu \nu}=\partial_{\mu} \mathbf{B}_{\nu}-\partial_{\nu} \mathbf{B}_{\mu}$ is the field strength tensor of magnetic field $\mathbf{B}_{\mu}$, which is dual to the field $\mathbf{a}_{\mu} \equiv\left(A_{\mu}^{3}, A_{\mu}^{8}\right)$, and $\mathbf{j}_{\nu}^{\mathrm{m}}=\partial_{\mu} \tilde{\mathbf{f}}_{\mu \nu}^{\mathrm{m}}$. A minor nontriviality, one meets further w.r.t. the simplest $S U(2)$-case, is the necessity to take into account the fact that monopole charges are distributed over the lattice defined by the root vectors, which have the form

$$
\mathbf{e}_{1}=(1,0), \mathbf{e}_{2}=\left(-\frac{1}{2},-\frac{\sqrt{3}}{2}\right), \mathbf{e}_{3}=\left(-\frac{1}{2}, \frac{\sqrt{3}}{2}\right) .
$$

These vectors naturally appear within the Cartan decomposition of the original set of gluonic fields as the structural constants in the commutation relations between the diagonal and so-called step (raising and lowering) operators. The collective current of $N$ monopole Cooper pairs then reads

$$
\mathbf{j}_{\mu}^{\mathrm{m}(N)}(x)=2 g_{m} \sum_{n=1}^{N} \sum_{a=1}^{3} \mathbf{e}_{a} \oint d x_{\mu}^{(a) n}(s) \delta\left(x-x^{(a) n}(s)\right) .
$$

As far as the average over the currents is concerned, it has the form

$$
\begin{gathered}
\left\langle\exp \left(i \int d^{4} x \mathbf{B}_{\mu} \mathbf{j}_{\mu}^{\mathrm{m}}\right)\right\rangle_{\mathbf{j}_{\mu}^{\mathrm{m}}}=\prod_{a=1}^{3}\left\{1+\sum_{N=1}^{\infty} \frac{1}{N !}\left[\prod_{n=1}^{N} \int_{0}^{+\infty} \frac{d s_{n}}{s_{n}} \mathrm{e}^{4 \lambda \eta^{2} s_{n}} \int_{u^{(a)}(0)=u^{(a)}\left(s_{n}\right)} D u^{(a)}\left(s_{n}^{\prime}\right)\right] \times\right. \\
\times \exp \left[\sum_{l=1}^{N} \int_{0}^{s_{l}} d s_{l}^{\prime}\left(-\frac{1}{4}\left(\dot{u}^{(a)}\left(s_{l}^{\prime}\right)\right)^{2}+2 i g_{m} \dot{u}_{\mu}^{(a)}\left(s_{l}^{\prime}\right) \mathbf{e}_{a} \mathbf{B}_{\mu}\left(u^{(a)}\left(s_{l}^{\prime}\right)\right)\right)-\right. \\
\left.\left.-4 \lambda \sum_{l, k=1}^{N} \int_{0}^{s_{l}} d s_{l}^{\prime} \int_{0}^{s_{k}} d s_{k}^{\prime \prime} \delta\left[u^{(a)}\left(s_{l}^{\prime}\right)-u^{(a)}\left(s_{k}^{\prime \prime}\right)\right]\right]\right\}= \\
=\int \mathcal{D} \Phi_{a} \mathcal{D} \Phi_{a}^{*} \exp \left\{-\int d^{4} x \sum_{a=1}^{3}\left[\frac{1}{2}\left|\left(\partial_{\mu}-2 i g_{m} \mathbf{e}_{a} \mathbf{B}_{\mu}\right) \Phi_{a}\right|^{2}+\lambda\left(\left|\Phi_{a}\right|^{2}-\eta^{2}\right)^{2}\right]\right\}
\end{gathered}
$$

where the vector $u_{\mu}^{(a)}\left(s_{n}^{\prime}\right)$ parametrizes the same contour as the vector $x_{\mu}^{(a) n}(s)$. Finally, it is worth noting that since monopoles are distributed over the root lattice, whose vectors are related to each other by the condition $\sum_{a=1}^{3} \mathbf{e}_{a}=0$, the dual Higgs fields $\Phi_{a}$ 's are also not completely independent of each other. In Ref. [8], it was argued that the relevant constraint for these fields reads $\sum_{a=1}^{3} \theta_{a}=0$. Taking this into account we arrive at the following partition function describing an effective $[U(1)]^{2}$ magnetically gauge-invariant AP theory of the $S U(3)$-gluodynamics [8]:

$$
\begin{gathered}
\mathcal{Z}=\int\left|\Phi_{a}\right| \mathcal{D}\left|\Phi_{a}\right| \mathcal{D} \theta_{a} \mathcal{D} \mathbf{B}_{\mu} \delta\left(\sum_{a=1}^{3} \theta_{a}\right) \times \\
\times \exp \left\{-\int d^{4} x\left[\frac{1}{4} \mathbf{F}_{\mu \nu}^{2}+\sum_{a=1}^{3}\left[\frac{1}{2}\left|\left(\partial_{\mu}-2 i g_{m} \mathbf{e}_{a} \mathbf{B}_{\mu}\right) \Phi_{a}\right|^{2}+\lambda\left(\left|\Phi_{a}\right|^{2}-\eta^{2}\right)^{2}\right]\right]\right\},
\end{gathered}
$$

where $\Phi_{a}=\left|\Phi_{a}\right| \mathrm{e}^{i \theta_{a}}$. 


\subsection{Bilocal Electric Field Strength Correlators}

\subsection{1 $S U(2)$-case}

In order to investigate bilocal cumulants of electric field strengths in the models (6) and (11), it is necessary to extend them by external electrically charged test particles (i.e. particles, charged w.r.t. the Cartan subgroup of the original $S U(2)$ - or $S U(3)$-group). For brevity, we shall call these particles simply "quarks". In the $S U(2)$-case, such an extension can be performed by adding to the action (2) the term $i \int d^{4} x a_{\mu} j_{\mu}^{\mathrm{e}}$ with $j_{\mu}^{\mathrm{e}}(x) \equiv g_{C} \oint_{C} d x_{\mu}(s) \delta(x-x(s))$ standing for the conserved electric current of a quark, which moves along a certain closed contour $C$. Then, performing the dualization of the so-extended action and summing up over monopole currents according to Eq. (4), we arrive at Eq. (6) with $F_{\mu \nu}$ replaced by $F_{\mu \nu}+F_{\mu \nu}^{\mathrm{e}}$. Here, $F_{\mu \nu}^{\mathrm{e}}$ stands for the field strength tensor generated by quarks according to the equation $\partial_{\mu} \tilde{F}_{\mu \nu}^{\mathrm{e}}=j_{\nu}^{\mathrm{e}}$. A solution to this equation reads $F_{\mu \nu}^{\mathrm{e}}=-g \tilde{\Sigma}_{\mu \nu}^{\mathrm{e}}$, where $\Sigma_{\mu \nu}^{\mathrm{e}}(x) \equiv \int_{\Sigma^{\mathrm{e}}} d \sigma_{\mu \nu}(\bar{x}(\xi)) \delta(x-\bar{x}(\xi))$ is the so-called vorticity tensor current defined at an arbitrary surface $\Sigma^{\mathrm{e}}$ (which is just the world sheet of an open dual Nielsen-Olesen string), bounded by the contour $C$.

From now on, we shall be interested in the London limit, $\lambda \rightarrow \infty$, of the theories (6) and (11), where they admit an exact string representation. In that limit, the partition function of the theory (6) with external quarks reads

$$
\mathcal{Z}=\int \mathcal{D} B_{\mu} \mathcal{D} \theta^{\text {sing. }} \mathcal{D} \theta^{\text {reg. }} \exp \left\{-\int d^{4} x\left[\frac{1}{4}\left(F_{\mu \nu}+F_{\mu \nu}^{\mathrm{e}}\right)^{2}+\frac{\eta^{2}}{2}\left(\partial_{\mu} \theta-2 g_{m} B_{\mu}\right)^{2}\right]\right\} .
$$

In Eq. (12), we have performed a decomposition of the phase of the dual Higgs field $\theta=\theta^{\text {sing. }}+\theta^{\text {reg. }}$, where the multivalued field $\theta^{\text {sing. }}(x)$ describes a certain configuration of the dual strings and obeys the equation 22

$$
\varepsilon_{\mu \nu \lambda \rho} \partial_{\lambda} \partial_{\rho} \theta^{\text {sing. }}(x)=2 \pi \Sigma_{\mu \nu}(x) .
$$

Here, $\Sigma_{\mu \nu}$ stands for the vorticity tensor current, defined at the world sheet $\Sigma$ of a closed dual string, parametrized by the vector $x_{\mu}(\xi)$. On the other hand, the field $\theta^{\text {reg. }}(x)$ describes simply a singlevalued fluctuation around the above mentioned string configuration.

The string representation of the theory (12) can be derived similarly to Ref. [22], where this has been done for a model with a global $U(1)$-symmetry. One gets

$$
\mathcal{Z}=\int \mathcal{D} x_{\mu}(\xi) \mathcal{D} h_{\mu \nu} \exp \left\{-\int d^{4} x\left[\frac{1}{12 \eta^{2}} H_{\mu \nu \lambda}^{2}+g_{m}^{2} h_{\mu \nu}^{2}+i \pi h_{\mu \nu} \hat{\Sigma}_{\mu \nu}\right]\right\}
$$

where $\hat{\Sigma}_{\mu \nu} \equiv 4 \Sigma_{\mu \nu}^{\mathrm{e}}-\Sigma_{\mu \nu}$, and $H_{\mu \nu \lambda} \equiv \partial_{\mu} h_{\nu \lambda}+\partial_{\lambda} h_{\mu \nu}+\partial_{\nu} h_{\lambda \mu}$ is the field strength tensor of a massive antisymmetric tensor field $h_{\mu \nu}$ (the so-called Kalb-Ramond field [23]). This antisymmetric spin-1 tensor field emerged via some constraints from the integration over $\theta^{\text {reg. }}$ and represents the massive dual vector boson. As far as the integration over the world sheets of closed strings, $\mathcal{D} x_{\mu}(\xi)$, is concerned, it appeared from the integration over $\theta^{\text {sing. }}$ by virtue of Eq. (13), owing to which there exists a one-to-one correspondence between $\theta^{\text {sing. }}$ and $x_{\mu}(\xi)$. Physically this correspondence stems from the fact that the singularity of the phase of the dual Higgs field just takes place at the string world sheets. (Notice that since in what follows we shall be interested in effective actions rather than the integration measures, the Jacobian emerging during the change of the integration 
variables $\theta^{\text {sing. }} \rightarrow x_{\mu}(\xi)$, which has been evaluated in Ref. [24], will not be discussed below and is assumed to be included into the measure $\mathcal{D} x_{\mu}(\xi)$.)

Finally, the Gaussian integration over the field $h_{\mu \nu}$ in Eq. (14) leads to the following expression for the partition function (12):

$$
\begin{gathered}
\mathcal{Z}=\exp \left[-\frac{g^{2}}{2} \oint_{C} d x_{\mu} \oint_{C} d y_{\mu} D_{m}^{(4)}(x-y)\right] \times \\
\times \int \mathcal{D} x_{\mu}(\xi) \exp \left[-(\pi \eta)^{2} \int d^{4} x \int d^{4} y \hat{\Sigma}_{\mu \nu}(x) D_{m}^{(4)}(x-y) \hat{\Sigma}_{\mu \nu}(y)\right] .
\end{gathered}
$$

Here, $D_{m}^{(4)}(x) \equiv \frac{m}{4 \pi^{2}|x|} K_{1}(m|x|)$ is the propagator of the dual vector boson, whose mass $m$, generated by the Higgs mechanism, is equal to $2 g_{m} \eta$, and $K_{\nu}$ 's henceforth stand for the modified Bessel functions. The details of derivation of Eqs. (14) and (15) can be found e.g. in Ref. 113. Besides that review, the obtained string representation (15) has been discussed in various contexts in Refs. [10, 12, 17, 24, 25]. Clearly, the first exponential factor on the R.H.S. of Eq. (15) is the standard result, which can be obtained without accounting for the dual Nielsen-Olesen strings. Contrary to that, the integral over string world sheets on the R.H.S. of this equation stems just from the contribution of strings to the partition function and is the essence of the string representation. The respective string effective action describes both the interaction of the closed world sheets $\Sigma$ 's with the open world sheets $\Sigma^{\mathrm{e}}$ 's and self-interactions of these objects.

We are now in the position to discuss the bilocal correlator of electric field strengths in the model (12). Indeed, owing to the Stokes theorem, such an extended partition function (which is actually nothing else, but the Wilson loop of a test quark) can be written as $\left\langle\exp \left(-\frac{i g}{2} \int d^{4} x \Sigma_{\mu \nu}^{\mathrm{e}} f_{\mu \nu}\right)\right\rangle_{a_{\mu}, j_{\mu}^{\mathrm{m}}}$, where $\langle\ldots\rangle_{a_{\mu}, j_{\mu}^{\mathrm{m}}} \equiv\left\langle\int \mathcal{D} a_{\mu} \exp \left(-S_{\text {eff. }}\left[a_{\mu}, f_{\mu \nu}^{\mathrm{m}}\right]\right)(\ldots)\right\rangle_{j_{\mu}^{\mathrm{m}}}$ with $S_{\text {eff. }}$ and $\langle\ldots\rangle_{j_{\mu}^{\mathrm{m}}}$ given by Eqs. (2) and (4), respectively. Applying to this expression the cumulant expansion, we have in the bilocal approximation:

$$
\mathcal{Z} \simeq \exp \left[-\frac{g^{2}}{8} \int d^{4} x \int d^{4} y \Sigma_{\mu \nu}^{\mathrm{e}}(x) \Sigma_{\lambda \rho}^{\mathrm{e}}(y)\left\langle\left\langle f_{\mu \nu}(x) f_{\lambda \rho}(y)\right\rangle\right\rangle_{a_{\mu}, j_{\mu}^{\mathrm{m}}}\right] .
$$

Following the SVM, let us parametrize the bilocal cumulant by the two Lorentz structures similarly to Eq. (1):

$$
\begin{gathered}
\left\langle\left\langle f_{\mu \nu}(x) f_{\lambda \rho}(0)\right\rangle\right\rangle_{a_{\mu}, j_{\mu}^{\mathrm{m}}}=\left(\delta_{\mu \lambda} \delta_{\nu \rho}-\delta_{\mu \rho} \delta_{\nu \lambda}\right) \mathcal{D}\left(x^{2}\right)+ \\
+\frac{1}{2}\left[\partial_{\mu}\left(x_{\lambda} \delta_{\nu \rho}-x_{\rho} \delta_{\nu \lambda}\right)+\partial_{\nu}\left(x_{\rho} \delta_{\mu \lambda}-x_{\lambda} \delta_{\mu \rho}\right)\right] \mathcal{D}_{1}\left(x^{2}\right) .
\end{gathered}
$$

Owing to the Stokes theorem, Eq. (17) yields

$$
\mathcal{Z} \simeq \exp \left\{-\frac{1}{8} \int d^{4} x \int d^{4} y\left[2 g^{2} \Sigma_{\mu \nu}^{\mathrm{e}}(x) \Sigma_{\mu \nu}^{\mathrm{e}}(y) \mathcal{D}\left((x-y)^{2}\right)+j_{\mu}^{\mathrm{e}}(x) j_{\mu}^{\mathrm{e}}(y) G\left((x-y)^{2}\right)\right]\right\}
$$

where

$$
G\left(x^{2}\right) \equiv \int_{x^{2}}^{+\infty} d \lambda \mathcal{D}_{1}(\lambda)
$$


On the other hand, Eq. (18) should coincide with Eq. (15) divided by $\mathcal{Z}\left[\Sigma_{\mu \nu}^{\mathrm{e}}=0\right]$ (which is just the standard normalization condition, encoded in the integration measures), i.e. it reads

$$
\begin{aligned}
\mathcal{Z}=\exp & \left\{-\int d^{4} x \int d^{4} y D_{m}^{(4)}(x-y)\left[(4 \pi \eta)^{2} \Sigma_{\mu \nu}^{\mathrm{e}}(x) \Sigma_{\mu \nu}^{\mathrm{e}}(y)+\frac{1}{2} j_{\mu}^{\mathrm{e}}(x) j_{\mu}^{\mathrm{e}}(y)\right]\right\} \times \\
\times & \left\langle\exp \left[8(\pi \eta)^{2} \int d^{4} x \int d^{4} y D_{m}^{(4)}(x-y) \Sigma_{\mu \nu}^{\mathrm{e}}(x) \Sigma_{\mu \nu}(y)\right]\right\rangle_{x_{\mu}(\xi)}
\end{aligned}
$$

where

$$
\langle\ldots\rangle_{x_{\mu}(\xi)} \equiv \frac{\int \mathcal{D} x_{\mu}(\xi)(\ldots) \exp \left[-(\pi \eta)^{2} \int d^{4} x \int d^{4} y \Sigma_{\mu \nu}(x) D_{m}^{(4)}(x-y) \Sigma_{\mu \nu}(y)\right]}{\int \mathcal{D} x_{\mu}(\xi) \exp \left[-(\pi \eta)^{2} \int d^{4} x \int d^{4} y \Sigma_{\mu \nu}(x) D_{m}^{(4)}(x-y) \Sigma_{\mu \nu}(y)\right]}
$$

As it has already been discussed in the Introduction, in the case of zero temperature under study, dual Nielsen-Olesen strings, one should average over in Eq. (21), form virtual bound states of vortex loops. The typical areas $|\Sigma|$ 's of those are very small, and in the leading approximation can be disregarded w.r.t. the area $\left|\Sigma^{\mathrm{e}}\right|$ of the world sheet of the long open string, which confines a test quark. Owing to this, the exponential factor, which should be averaged over vortex loops on the R.H.S. of Eq. (20), can be disregarded w.r.t. the first exponential factor in this equation, as well. Then, the comparison of the latter one with Eq. (18) straightforwardly yields for the function $\mathcal{D}$ the following expression

$$
\mathcal{D}\left(x^{2}\right)=\frac{m^{3}}{4 \pi^{2}} \frac{K_{1}(m|x|)}{|x|}
$$

whereas for the function $\mathcal{D}_{1}$ we get the equation $G\left(x^{2}\right)=4 D_{m}^{(4)}(x)$, which leads to:

$$
\mathcal{D}_{1}\left(x^{2}\right)=\frac{m}{2 \pi^{2} x^{2}}\left[\frac{K_{1}(m|x|)}{|x|}+\frac{m}{2}\left(K_{0}(m|x|)+K_{2}(m|x|)\right)\right] .
$$

We see that in the IR limit $|x| \gg \frac{1}{m}$, the asymptotic behaviours of the coefficient functions (22) and (23) are given by

$$
\mathcal{D} \longrightarrow \frac{m^{4}}{4 \sqrt{2} \pi^{\frac{3}{2}}} \frac{\mathrm{e}^{-m|x|}}{(m|x|)^{\frac{3}{2}}}
$$

and

$$
\mathcal{D}_{1} \longrightarrow \frac{m^{4}}{2 \sqrt{2} \pi^{\frac{3}{2}}} \frac{\mathrm{e}^{-m|x|}}{(m|x|)^{\frac{5}{2}}}
$$

For bookkeeping purposes, let us also present the asymptotic behaviours of these functions in the opposite case, $|x| \ll \frac{1}{m}$. Those read

$$
\mathcal{D} \longrightarrow \frac{m^{2}}{4 \pi^{2} x^{2}}
$$

and

$$
\mathcal{D}_{1} \longrightarrow \frac{1}{\pi^{2}|x|^{4}}
$$


One can now see that according to the lattice data [3, 4], the asymptotic behaviours (24) and (25) are very similar to the IR ones of the nonperturbative parts of the functions $D$ and $D_{1}$, which parametrize the bilocal cumulant (1) in the case of QCD. In particular, both functions decrease exponentially, and the function $\mathcal{D}$ is much larger than the function $\mathcal{D}_{1}$ due to the preexponential power-like behaviour. We also see that the rôle of the correlation length of the vacuum, $T_{g}$, is the SVM, i.e. the distance at which the functions $D$ and $D_{1}$ decrease, is played in the model (12) by the inverse mass of the dual vector boson, $\frac{1}{m}$. Moreover, the UV asymptotic behaviours (26) and (27) also parallel the results of the SVM in QCD to the lowest order of perturbation theory. Namely, at such distances the function $D_{1}$ also behaves as $\frac{1}{|x|^{4}}$ due to the one-gluon-exchange contribution. As far as the function $D$ is concerned, it vanishes to the leading order of perturbation theory. Although this is not the case in our model (whose UV features are far from those of the asymptotically free QCD), the $\mathcal{D}_{1}$-asymptotics (27) is nevertheless really much larger than that of the $\mathcal{D}$-function, given by Eq. (26).

Hence we see that within the approximation when the contribution of vortex loops to the partition function (20) is disregarded completely, the bilocal approximation to the SVM is an exact result in the theory (12), i.e. all the cumulants of the orders higher than the second one vanish. Higher cumulants naturally appear upon performing in Eq. (20) the average (21) over vortex loops. However, this average yields important modifications already on the level of the bilocal cumulant. Namely, as we shall see in the next Section, it modifies the classical expressions (22) and (23).

\subsection{2 $S U(3)$-case}

Let us now turn ourselves to the bilocal cumulant of electric field strength tensors in the London limit of AP $S U(3)$-gluodynamics, where the partition function (11) of this theory takes the form

$$
\mathcal{Z}=\int \mathcal{D} \mathbf{B}_{\mu} \mathcal{D} \theta_{a}^{\text {sing. }} \mathcal{D} \theta_{a}^{\text {reg. }} \delta\left(\sum_{a=1}^{3} \theta_{a}\right) \exp \left\{-\int d^{4} x\left[\frac{1}{4} \mathbf{F}_{\mu \nu}^{2}+\frac{\eta^{2}}{2} \sum_{a=1}^{3}\left(\partial_{\mu} \theta_{a}-2 g_{m} \mathbf{e}_{a} \mathbf{B}_{\mu}\right)^{2}\right]\right\}
$$

Similarly to the $S U(2)$-case, in the model under study there exist dual Nielsen-Olesen-type strings. Due to that, in Eq. (28) we have again decomposed the total phases of the dual Higgs fields into

the multivalued and singlevalued parts, $\theta_{a}=\theta_{a}^{\text {sing. }}+\theta_{a}^{\text {reg. }}$. Here, the multivalued parts $\theta_{a}^{\text {sing.' }} \mathrm{s}$ describe a given configuration of the dual strings of three types. They are related to the world sheets $\Sigma_{a}$ 's of these strings via the equations

$$
\varepsilon_{\mu \nu \lambda \rho} \partial_{\lambda} \partial_{\rho} \theta_{a}^{\text {sing. }}(x)=2 \pi \Sigma_{\mu \nu}^{a}(x) \equiv 2 \pi \int_{\Sigma_{a}} d \sigma_{\mu \nu}\left(x_{a}(\xi)\right) \delta\left(x-x_{a}(\xi)\right),
$$

where $x_{a} \equiv x_{\mu}^{a}(\xi)$ is a four-vector parametrizing the world sheet $\Sigma_{a}$.

An external quark of a certain colour $c=R, B, G$ (red, blue, green, respectively) can be introduced into the theory under study by adding to the initial action (可) the interaction term $i \mathbf{Q}^{(c)} \int d^{4} x \mathbf{a}_{\mu} j_{\mu}^{\mathrm{e}}$, where the vectors of colour charges read

$$
\mathbf{Q}^{(R)}=\left(\frac{1}{2}, \frac{1}{2 \sqrt{3}}\right), \mathbf{Q}^{(B)}=\left(-\frac{1}{2}, \frac{1}{2 \sqrt{3}}\right), \mathbf{Q}^{(G)}=\left(0,-\frac{1}{\sqrt{3}}\right) .
$$

These vectors are just the weights of the representation 3 of ${ }^{*} S U(3)$. In another words, those are nothing else, but the charges of quarks w.r.t. the Cartan subgroup $[U(1)]^{2}$, i.e. for every $c$, the 
components of $\mathbf{Q}^{(c)}$ are just the charges of a quark of the colour $c$ w.r.t. the diagonal gluons $A_{\mu}^{3}$ and $A_{\mu}^{8}$.

Applying further the Stokes theorem and the cumulant expansion in the bilocal approximation, we get for the partition function of the theory (28) with an external quark of the colour $c$ the following expression:

$$
\mathcal{Z}_{c} \simeq \exp \left[-\frac{g^{2}}{8} Q^{(c) i} Q^{(c) j} \int d^{4} x \int d^{4} y \Sigma_{\mu \nu}^{\mathrm{e}}(x) \Sigma_{\lambda \rho}^{\mathrm{e}}(y)\left\langle\left\langle f_{\mu \nu}^{i}(x) f_{\lambda \rho}^{j}(y)\right\rangle\right\rangle_{\mathbf{a}_{\mu}, \mathbf{j}_{\mu}^{\mathrm{m}}}\right] .
$$

Here, $i, j=1,2$ denote the $[U(1)]^{2}$-indices, referring to the Cartan generators $\left(T^{3}, T^{8}\right)$, and the average is defined as $\langle\ldots\rangle_{\mathbf{a}_{\mu}, \mathbf{j}_{\mu}^{\mathrm{m}}} \equiv\left\langle\int \mathcal{D} \mathbf{a}_{\mu} \exp \left(-S_{\text {eff. }}\left[\mathbf{a}_{\mu}, \mathbf{f}_{\mu \nu}^{\mathrm{m}}\right]\right)(\ldots)\right\rangle_{\mathbf{j}_{\mu}^{\mathrm{m}}}$, where $S_{\text {eff. }}$ and $\langle\ldots\rangle_{\mathbf{j}_{\mu}^{\mathrm{m}}}$ are given by Eqs. (7) and (10), respectively. Upon the SVM-inspired parametrization of the bilocal cumulant,

$$
\begin{aligned}
& \left\langle\left\langle f_{\mu \nu}^{i}(x) f_{\lambda \rho}^{j}(0)\right\rangle\right\rangle_{\mathbf{a}_{\mu}, \mathbf{j}_{\mu}^{\mathrm{m}}}=\delta^{i j}\left\{\left(\delta_{\mu \lambda} \delta_{\nu \rho}-\delta_{\mu \rho} \delta_{\nu \lambda}\right) \hat{D}\left(x^{2}\right)+\right. \\
& \left.+\frac{1}{2}\left[\partial_{\mu}\left(x_{\lambda} \delta_{\nu \rho}-x_{\rho} \delta_{\nu \lambda}\right)+\partial_{\nu}\left(x_{\rho} \delta_{\mu \lambda}-x_{\lambda} \delta_{\mu \rho}\right)\right] \hat{D}_{1}\left(x^{2}\right)\right\},
\end{aligned}
$$

we can write for Eq. (30) the following expression:

$$
\mathcal{Z}_{c} \simeq \exp \left\{-\frac{1}{24} \int d^{4} x \int d^{4} y\left[2 g^{2} \Sigma_{\mu \nu}^{\mathrm{e}}(x) \Sigma_{\mu \nu}^{\mathrm{e}}(y) \hat{D}\left((x-y)^{2}\right)+j_{\mu}^{\mathrm{e}}(x) j_{\mu}^{\mathrm{e}}(y) \hat{G}\left((x-y)^{2}\right)\right]\right\} .
$$

Here, we have denoted by $\hat{G}$ the same function as (19), but with the replacement $\mathcal{D}_{1} \rightarrow \hat{D}_{1}$, and used the fact that for every $c,\left(\mathbf{Q}^{(c)}\right)^{2}=\frac{1}{3}$.

On the other hand, one can derive the string representation for the partition function $\mathcal{Z}_{c}$. Indeed, the dualization of the action (7) with the term $i \mathbf{Q}^{(c)} \int d^{4} x \mathbf{a}_{\mu} j_{\mu}^{\mathrm{e}}$ added, leads to Eq. (8) with $\mathbf{F}_{\mu \nu}$ replaced by $\mathbf{F}_{\mu \nu}+\mathbf{F}_{\mu \nu}^{(c)}$. Here, $\mathbf{F}_{\mu \nu}^{(c)}$ stands for the field strength tensor of a test quark of the colour $c$, which obeys the equation $\partial_{\mu} \tilde{\mathbf{F}}_{\mu \nu}^{(c)}=\mathbf{Q}^{(c)} j_{\nu}^{\mathrm{e}}$ and thus can be written as $\mathbf{F}_{\mu \nu}^{(c)}=-g \mathbf{Q}^{(c)} \tilde{\Sigma}_{\mu \nu}^{\mathrm{e}}$. Next, the summation over the currents of monopole Cooper pairs in the sense of Eq. (10) yields Eq. (11) with the same extension of $\mathbf{F}_{\mu \nu}$. In the London limit under study, the string representation of this theory (see Refs. [11, 26] for details) reads

$$
\begin{gathered}
\mathcal{Z}_{c}=\int \mathcal{D} x_{\mu}^{a}(\xi) \delta\left(\sum_{a=1}^{3} \Sigma_{\mu \nu}^{a}\right) \times \\
\times \exp \left\{-\pi^{2} \int d^{4} x \int d^{4} y D_{m_{B}}^{(4)}(x-y)\left[\eta^{2} \bar{\Sigma}_{\mu \nu}^{a}(x) \bar{\Sigma}_{\mu \nu}^{a}(y)+\frac{1}{6 \pi^{2}} j_{\mu}^{\mathrm{e}}(x) j_{\mu}^{\mathrm{e}}(y)\right]\right\} .
\end{gathered}
$$

Here, $m_{B}=\sqrt{6} g_{m} \eta$ is the mass of the dual vector bosons, which they acquire due to the Higgs mechanism. We have also introduced the notation $\bar{\Sigma}_{\mu \nu}^{a} \equiv \Sigma_{\mu \nu}^{a}-2 s_{a}^{(c)} \Sigma_{\mu \nu}^{\mathrm{e}}$ with the following numbers $s_{a}^{(c)}$ 's: $s_{3}^{(R)}=s_{2}^{(B)}=s_{1}^{(G)}=0, s_{1}^{(R)}=s_{3}^{(B)}=s_{2}^{(G)}=-s_{2}^{(R)}=-s_{1}^{(B)}=-s_{3}^{(G)}=1$, which obey the relation $\mathbf{Q}^{(c)}=\frac{1}{3} \mathbf{e}_{a} s_{a}^{(c)}$. Taking into account that for every $c,\left(s_{a}^{(c)}\right)^{2}=2$, we eventually arrive at the following expression for the partition function ( $c f$. Eq. (20)):

$$
\mathcal{Z}_{c}=\exp \left\{-8 \pi^{2} \int d^{4} x \int d^{4} y D_{m_{B}}^{(4)}(x-y)\left[\eta^{2} \Sigma_{\mu \nu}^{\mathrm{e}}(x) \Sigma_{\mu \nu}^{\mathrm{e}}(y)+\frac{1}{48 \pi^{2}} j_{\mu}^{\mathrm{e}}(x) j_{\mu}^{\mathrm{e}}(y)\right]\right\} \times
$$




$$
\times\left\langle\exp \left[(2 \pi \eta)^{2} s_{a}^{(c)} \int d^{4} x \int d^{4} y \Sigma_{\mu \nu}^{a}(x) D_{m_{B}}^{(4)}(x-y) \Sigma_{\mu \nu}^{\mathrm{e}}(y)\right]\right\rangle_{x_{\mu}^{a}(\xi)}
$$

with the average over vortex loops having the form

$$
\langle\ldots\rangle_{x_{\mu}^{a}(\xi)} \equiv \frac{\int \mathcal{D} x_{\mu}^{a}(\xi) \delta\left(\sum_{a=1}^{3} \Sigma_{\mu \nu}^{a}\right)(\ldots) \exp \left[-(\pi \eta)^{2} \int d^{4} x \int d^{4} y \Sigma_{\mu \nu}^{a}(x) D_{m_{B}}^{(4)}(x-y) \Sigma_{\mu \nu}^{a}(y)\right]}{\int \mathcal{D} x_{\mu}^{a}(\xi) \delta\left(\sum_{a=1}^{3} \Sigma_{\mu \nu}^{a}\right) \exp \left[-(\pi \eta)^{2} \int d^{4} x \int d^{4} y \Sigma_{\mu \nu}^{a}(x) D_{m_{B}}^{(4)}(x-y) \Sigma_{\mu \nu}^{a}(y)\right]}
$$

Comparing now Eq. (32) with Eq. (34), we see that in the approximation of very small vortex loops, $\left|\Sigma^{a}\right| \ll\left|\Sigma^{\mathrm{e}}\right|$, the functions $\hat{D}$ and $D_{1}$ are given by Eqs. (22) and (23) with the replacement $m \rightarrow m_{B}$. Besides that, it is obvious that the bilocal cumulant (31) is nonvanishing only for the gluonic field strength tensors of the same kind, i.e. for $i=j=1$ or $i=j=2$. Hence, for these diagonal cumulants, the vacuum of the AP $S U(3)$-gluodynamics in the London limit does exhibit a nontrivial correlation length $T_{g}=\frac{1}{m_{B}}$.

\section{Electric Field Strength Correlators in the Gas of Vortex Loops}

\section{1 $S U(2)$-case}

To study the properties of vortex loops in the above considered theories, there is clearly no necessity to introduce external quarks. The field strength correlators can be studied afterwards, i.e. already after the summation over the grand canonical ensemble of vortex loops. Thus, let us first consider the theory (12) with $F_{\mu \nu}^{\mathrm{e}}=0$. Upon the derivation of the string representation of such a theory, we are then left with Eq. (14), where $\Sigma_{\mu \nu}^{\mathrm{e}}=0$. To study the grand canonical ensemble of vortex loops, it is necessary to replace $\Sigma_{\mu \nu}$ in Eq. (14) by the following expression:

$$
\Sigma_{\mu \nu}^{\mathrm{gas}}(x)=\sum_{i=1}^{N} n_{i} \int d \sigma_{\mu \nu}\left(x_{i}(\xi)\right) \delta\left(x-x_{i}(\xi)\right) .
$$

Here, $\xi \in[0,1] \times[0,1]$ is a $2 \mathrm{D}$-coordinate, and $n_{i}$ 's stand for winding numbers. In what follows, we shall restrict ourselves to the vortex loops possessing the minimal winding numbers, $n_{i}= \pm 1$. That is because, analogously to the 3D-case [14, 27], the energy of a single vortex loop is known to be a quadratic function of its flux, owing to which the existence of two vortex loops of a unit flux is more energetically favourable than the existence of one vortex loop of the double flux.

Then, taking into account that the gas of vortex loops is dilute, one can perform the summation over the grand canonical ensemble of these objects, which yields the following expression for the partition function [17]:

$$
\mathcal{Z}=\int \mathcal{D} h_{\mu \nu} \exp \left\{-\int d^{4} x\left[\frac{1}{12 \eta^{2}} H_{\mu \nu \lambda}^{2}+g_{m}^{2} h_{\mu \nu}^{2}-2 \zeta \cos \left(\frac{\left|h_{\mu \nu}\right|}{\Lambda^{2}}\right)\right]\right\} .
$$

Here $\left|h_{\mu \nu}\right| \equiv \sqrt{h_{\mu \nu}^{2}}$, and $\Lambda \equiv \sqrt{\frac{L}{\pi a^{3}}}$ is an UV momentum cutoff with $L$ and $a$ denoting the characteristic distances between vortex loops and their typical sizes, respectively. Clearly in the 
dilute gas approximation under study, $a \ll L$ and $\Lambda \gg a^{-1}$. Also in Eq. (36), $\zeta \propto \mathrm{e}^{-S_{0}}$ stands for the fugacity (Boltzmann factor) of a single vortex loop, which has the dimension (mass) ${ }^{4}$, with $S_{0}$ denoting the action of a single loop.

Note that the value of $S_{0}$ is approximately equal to $\sigma a^{2}$, where we have estimated the area of the vortex loop as $a^{2}$, and $\sigma$ stands for the string tension of the loop, i.e. its energy per unit area. This energy can be evaluated from the action standing in the arguments of the exponents on the R.H.S. of Eq. (21) by virtue of the results of Ref. [28] and reads

$$
\sigma=\frac{\eta^{2}}{2} \int d^{2} t \frac{K_{1}(|t|)}{|t|} \simeq \frac{\pi \eta^{2}}{2} \ln \left(\frac{\lambda}{g_{m}^{2}}\right)
$$

Here we have in the standard way [27] set for a characteristic small dimensionless quantity in the model under study the value $\frac{g_{m}}{\sqrt{\lambda}}$, which is of the order of the ratio of $m$ to the mass of the dual Higgs field. Moreover, it has been assumed that not only $\frac{\sqrt{\lambda}}{g_{m}} \gg 1$, but also $\ln \left(\frac{\sqrt{\lambda}}{g_{m}}\right) \gg 1$, i.e. the last equality on the R.H.S. of Eq. (37) is valid with the logarithmic accuracy. The physical origin of this logarithmic divergency is analogous to that, which takes place in 3D [14, 27] and is based on the fact that at the world sheet of a vortex loop the condensate of the dual Higgs field is destroyed, and the dual vector boson remains massless.

The square of the full mass of the field $h_{\mu \nu}$ following from Eq. (36) reads $M^{2}=m^{2}+m_{D}^{2} \equiv Q^{2} \eta^{2}$. Here, $m_{D}=\frac{2 \eta \sqrt{\zeta}}{\Lambda^{2}}$ is the additional contribution, emerging due to the screening of magnetic charge of the dual vector boson in the gas of electric vortex loops, and $Q=2 \sqrt{g_{m}^{2}+\frac{\zeta}{\Lambda^{4}}}$ is the full magnetic charge of the dual vector boson.

To study the correlation functions of vortex loops, it is convenient to represent the partition function (36) directly as an integral over these objects. This can be done by virtue of the following equality,

$$
\begin{gathered}
\exp \left\{-\int d^{4} x\left[\frac{1}{12 \eta^{2}} H_{\mu \nu \lambda}^{2}+g_{m}^{2} h_{\mu \nu}^{2}\right]\right\}= \\
=\int \mathcal{D} S_{\mu \nu} \exp \left\{-\left[(\pi \eta)^{2} \int d^{4} x \int d^{4} y S_{\mu \nu}(x) D_{m}^{(4)}(x-y) S_{\mu \nu}(y)+i \pi \int d^{4} x h_{\mu \nu} S_{\mu \nu}\right]\right\},
\end{gathered}
$$

in whose derivation it has been taken into account that $\partial_{\mu} h_{\mu \nu}=0$. Indeed, owing to the Hodge decomposition theorem, the Kalb-Ramond field can always be represented as follows: $h_{\mu \nu}=$ $\partial_{\mu} \varphi_{\nu}-\partial_{\nu} \varphi_{\mu}+\varepsilon_{\mu \nu \lambda \rho} \partial_{\lambda} \psi_{\rho}$. Clearly, in the original expression for the partition function,

$$
\mathcal{Z}=\left\langle\int \mathcal{D} h_{\mu \nu} \exp \left\{-\int d^{4} x\left[\frac{1}{12 \eta^{2}} H_{\mu \nu \lambda}^{2}+g_{m}^{2} h_{\mu \nu}^{2}-i \pi h_{\mu \nu} \Sigma_{\mu \nu}^{\mathrm{gas}}\right]\right\}\right\rangle_{\text {gas }}
$$

the field $\varphi_{\mu}$ decouples not only from $\Sigma_{\mu \nu}^{\text {gas }}$ (due to the conservation of the latter one), but also from $\psi_{\mu}$. The $\varphi_{\mu}$-field thus yields only an inessential determinant factor, which is not of our interest. Therefore this field can be disregarded, which proves the above statement.

Substituting now Eq. (38) into Eq. (36), we can integrate the field $h_{\mu \nu}$ out. This yields the desired representation for the partition function:

$$
\mathcal{Z}=\int \mathcal{D} S_{\mu \nu} \exp \left\{-\left[(\pi \eta)^{2} \int d^{4} x \int d^{4} y S_{\mu \nu}(x) D_{m}^{(4)}(x-y) S_{\mu \nu}(y)+V\left[S_{\mu \nu}\right]\right]\right\}
$$


where the effective potential of vortex loops, $V$, reads

$$
V\left[S_{\mu \nu}\right]=\int d^{4} x\left\{\pi \Lambda^{2}\left|S_{\mu \nu}\right| \ln \left[\frac{\pi \Lambda^{2}}{2 \zeta}\left|S_{\mu \nu}\right|+\sqrt{1+\left(\frac{\pi \Lambda^{2}}{2 \zeta}\left|S_{\mu \nu}\right|\right)^{2}}\right]-2 \zeta \sqrt{1+\left(\frac{\pi \Lambda^{2}}{2 \zeta}\left|S_{\mu \nu}\right|\right)^{2}}\right\} .
$$

It is straightforward to prove that the correlation functions of $S_{\mu \nu}$ 's, calculated by virtue of the representation (40), are nothing else, but the correlation functions of vortex loops in the gas. This can be seen in the following way. Let us integrate the $h_{\mu \nu}$-field out of the initial expression (39) for the partition function of the gas of vortex loops. This yields (cf. Eq. (21)):

$$
\mathcal{Z}=\left\langle\exp \left[-(\pi \eta)^{2} \int d^{4} x \int d^{4} y \Sigma_{\mu \nu}^{\mathrm{gas}}(x) D_{m}^{(4)}(x-y) \Sigma_{\mu \nu}^{\mathrm{gas}}(y)\right]\right\rangle_{\text {gas }} .
$$

This equation is now perfect to involve $S_{\mu \nu}$ 's and demonstrate that their correlation functions are indeed equal to those of vortex loops. After that, the $h_{\mu \nu}$-dependence can be restored back, so that we shall eventually again arrive at Eq. (36) with the substitution (38). In order to involve $S_{\mu \nu}$ 's, let us rewrite Eq. (42) as follows:

$$
\begin{gathered}
\mathcal{Z}=1+\sum_{N=1}^{\infty} \frac{\zeta^{N}}{N !} \times \\
\times\left\langle\int \mathcal{D} S_{\mu \nu} \delta\left(S_{\mu \nu}-\Sigma_{\mu \nu}^{\text {gas }}\right) \exp \left[-(\pi \eta)^{2} \int d^{4} x \int d^{4} y S_{\mu \nu}(x) D_{m}^{(4)}(x-y) S_{\mu \nu}(y)\right]\right\rangle_{\left\{x_{i}(\xi)\right\}_{i=1}^{N}} .
\end{gathered}
$$

The average here reads

$$
\langle\mathcal{O}\rangle_{\left\{x_{i}(\xi)\right\}_{i=1}^{N}} \equiv \prod_{i=1}^{N} \int d^{4} y_{i} \int \mathcal{D} z_{i}(\xi) \mu\left[z_{i}\right] \sum_{n_{i}= \pm 1} \mathcal{O}
$$

In this formula, the vector $y_{i}$ describes the position of the world sheet of the $i$-th vortex loop 5 , whereas the vector $z_{i}(\xi)$ describes its shape, i.e. $x_{i}(\xi)=y_{i}+z_{i}(\xi), y_{i}=\int d^{2} \xi x_{i}(\xi)$. We have also denoted by $\mu$ a certain rotation- and translation invariant measure of integration over shapes of the world sheets of vortex loops. Note that it was just this average, which in the dilute gas approximation led from Eq. (39) to Eq. (36) (see Ref. 17] for details).

From the $\delta$-function in Eq. (43) it is now clearly seen that the correlation functions of $S_{\mu \nu}$ 's are indeed equal to those of vortex loops. One can further represent this $\delta$-function as an integral over the Lagrange multiplier, whose rôle, as we shall see immediately below, is just played by the Kalb-Ramond field:

$$
\delta\left(S_{\mu \nu}-\Sigma_{\mu \nu}^{\mathrm{gas}}\right)=\int \mathcal{D} h_{\mu \nu} \exp \left[-i \pi \int d^{4} x h_{\mu \nu}\left(S_{\mu \nu}-\Sigma_{\mu \nu}^{\mathrm{gas}}\right)\right] .
$$

Indeed, normalizing the measure $\mathcal{D} h_{\mu \nu}$ by the condition f

$$
\int \mathcal{D} h_{\mu \nu} \mathcal{D} S_{\mu \nu} \exp \left[-(\pi \eta)^{2} \int d^{4} x \int d^{4} y S_{\mu \nu}(x) D_{m}^{(4)}(x-y) S_{\mu \nu}(y)-i \pi \int d^{4} x h_{\mu \nu} S_{\mu \nu}\right]=1,
$$

\footnotetext{
${ }^{3}$ For brevity, we omit the Lorentz index.

${ }^{4}$ Owing to Eq. (38), this condition can be rewritten simply as $\int \mathcal{D} h_{\mu \nu} \exp \left\{-\int d^{4} x\left[\frac{1}{12 \eta^{2}} H_{\mu \nu \lambda}^{2}+g_{m}^{2} h_{\mu \nu}^{2}\right]\right\}=1$.
} 
we get

$$
\begin{aligned}
\mathcal{Z}=\int \mathcal{D} S_{\mu \nu} \mathcal{D} h_{\mu \nu} & \exp \exp \left\{-\left[(\pi \eta)^{2} \int d^{4} x \int d^{4} y S_{\mu \nu}(x) D_{m}^{(4)}(x-y) S_{\mu \nu}(y)+\right.\right. \\
& \left.\left.+i \pi \int d^{4} x h_{\mu \nu} S_{\mu \nu}-2 \zeta \cos \left(\frac{\left|h_{\mu \nu}\right|}{\Lambda^{2}}\right)\right]\right\}
\end{aligned}
$$

This is just Eq. (36) with the substitution (38), which completes our proof.

The correlation functions of vortex loops can now be calculated in the approximation when the loop gas is sufficiently dilute, namely its density obeys the inequality $\left|S_{\mu \nu}\right| \ll \frac{\zeta}{\Lambda^{2}}$. Within this approximation, the potential (41) becomes a simple quadratic functional of $S_{\mu \nu}$ 's, and the generating functional for the correlators of vortex loops takes a simple Gaussian form. It reads

$$
\begin{gathered}
\mathcal{Z}\left[J_{\mu \nu}\right]=\frac{1}{\mathcal{Z}[0]} \int \mathcal{D} S_{\mu \nu} \exp \left\{-\left[(\pi \eta)^{2} \int d^{4} x \int d^{4} y S_{\mu \nu}(x) D_{m}^{(4)}(x-y) S_{\mu \nu}(y)+\right.\right. \\
\left.\left.+\int d^{4} x\left(-2 \zeta+\frac{\pi^{2} \Lambda^{4}}{4 \zeta} S_{\mu \nu}^{2}+J_{\mu \nu} S_{\mu \nu}\right)\right]\right\}=\exp \left[-\int d^{4} x \int d^{4} y J_{\mu \nu}(x) \mathcal{G}(x-y) J_{\mu \nu}(y)\right]
\end{gathered}
$$

where

$$
\mathcal{G}(x) \equiv \frac{\zeta}{\pi^{2} \Lambda^{4}}\left(\partial^{2}-m^{2}\right) D_{M}^{(4)}(x) .
$$

Next, since $\partial_{\mu} \Sigma_{\mu \nu}^{\text {gas }}=0$, the $\delta$-function in Eq. (43) requires that $\partial_{\mu} S_{\mu \nu}=0$ as well. The Hodge decomposition theorem then leads to the following representation for $S_{\mu \nu}: S_{\mu \nu}=\varepsilon_{\mu \nu \lambda \rho} \partial_{\lambda} \varphi_{\rho}$. Owing to this fact and the same theorem, the coupling $\int d^{4} x J_{\mu \nu} S_{\mu \nu}$ will be nonvanishing only provided that $J_{\mu \nu}=\varepsilon_{\mu \nu \lambda \rho} \partial_{\lambda} I_{\rho}$. This coupling then reads $2 \int d^{4} x I_{\mu} T_{\mu \nu} \varphi_{\nu}$, where $T_{\mu \nu}(x) \equiv \partial_{\mu}^{x} \partial_{\nu}^{x}-\delta_{\mu \nu} \partial^{x 2}$. On the other hand, substituting the above representation for $J_{\mu \nu}$ into the R.H.S. of Eq. (46), we have

$$
\mathcal{Z}\left[J_{\mu \nu}\right]=\exp \left[-2 \int d^{4} x \int d^{4} y I_{\mu}(x) I_{\nu}(y) T_{\mu \nu}(x) \mathcal{G}(x-y)\right] .
$$

Thus, varying $\mathcal{Z}\left[J_{\mu \nu}\right]$ twice w.r.t. $I_{\mu}$ and setting then $I_{\mu}=0$, we get

$$
T_{\mu \nu}(x) T_{\lambda \rho}(y)\left\langle\varphi_{\nu}(x) \varphi_{\rho}(y)\right\rangle=-T_{\mu \lambda}(x) \mathcal{G}(x-y) .
$$

Due to the rotation- and translation invariance of space-time, it is natural to seek for $\left\langle\varphi_{\nu}(x) \varphi_{\rho}(y)\right\rangle$ in the form of the following Ansatz: $\delta_{\nu \rho} g(x-y)$. This yields the equation $\partial^{2} g=\mathcal{G}$, whose solution reads

$$
g(x)=-\frac{\zeta}{\pi^{2} \Lambda^{4}}\left(\partial^{x 2}-m^{2}\right) \int d^{4} y D_{0}^{(4)}(x-y) D_{M}^{(4)}(y)
$$

where $\left.D_{0}^{(4)}(x) \equiv D_{m}^{(4)}(x)\right|_{m=0}=\frac{1}{4 \pi^{2} x^{2}}$. The last integral can obviously be rewritten as

$$
\int d^{4} z D_{0}^{(4)}(z) D_{M}^{(4)}(z-x)
$$

As we shall see below, it will be necessary to know the more general expression, namely that for the integral 


$$
\int d^{4} z D_{m}^{(4)}(z) D_{M}^{(4)}(z-x)
$$

Its calculation is outlined in the Appendix, and the result reads

$$
\frac{1}{m_{D}^{2}}\left(D_{m}^{(4)}(x)-D_{M}^{(4)}(x)\right) .
$$

Note that according to Eq. (49), Eq. (50) should be invariant w.r.t. the interchange $m \leftrightarrow M$. By noting that during this interchange $m_{D}^{2}$ changes its sign, one can see that this invariance really holds.

Setting now in Eq. (50) $m=0$, we get $\frac{1}{m_{D}^{2}}\left(D_{0}^{(4)}(x)-D_{m_{D}}^{(4)}(x)\right)$, which yields for the desired integral (48) the same result with the substitution $m_{D} \rightarrow M$. Thus, the final expression for the function $g$ reads

$$
g(x)=\frac{\zeta}{\left(\pi M \Lambda^{2}\right)^{2}}\left(\partial^{2}-m^{2}\right)\left(D_{M}^{(4)}(x)-D_{0}^{(4)}(x)\right) .
$$

The desired correlator of $S_{\mu \nu}$ 's has the form

$$
\left\langle S_{\mu \nu}(x) S_{\lambda \rho}(y)\right\rangle=\varepsilon_{\mu \nu \alpha \beta} \varepsilon_{\lambda \rho \gamma \sigma} \partial_{\alpha}^{x} \partial_{\gamma}^{y}\left\langle\varphi_{\beta}(x) \varphi_{\sigma}(y)\right\rangle
$$

and therefore

$$
\begin{gathered}
\left\langle S_{\mu \nu}(x) S_{\lambda \rho}(0)\right\rangle=-\varepsilon_{\mu \nu \alpha \beta} \varepsilon_{\lambda \rho \gamma \beta} \partial_{\alpha}^{x} \partial_{\gamma}^{x} g(x)= \\
=\left(\delta_{\lambda \nu} \delta_{\mu \rho}-\delta_{\nu \rho} \delta_{\mu \lambda}\right) \mathcal{G}(x)+\left(\delta_{\mu \lambda} \partial_{\rho} \partial_{\nu}+\delta_{\nu \rho} \partial_{\mu} \partial_{\lambda}-\delta_{\mu \rho} \partial_{\lambda} \partial_{\nu}-\delta_{\lambda \nu} \partial_{\mu} \partial_{\rho}\right) g(x),
\end{gathered}
$$

where it has been used that $\partial^{2} g(x)=\mathcal{G}(x)$.

This result can now straightforwardly be used for the calculation of the contribution of vortex loops to the bilocal cumulant (17). Indeed, applying to the average on the R.H.S. of Eq. (20) the cumulant expansion in the bilocal approximation, we get:

$$
\begin{gathered}
\mathcal{Z} \simeq \exp \left\{-\int d^{4} x \int d^{4} y D_{m}^{(4)}(x-y)\left[(4 \pi \eta)^{2} \Sigma_{\mu \nu}^{\mathrm{e}}(x) \Sigma_{\mu \nu}^{\mathrm{e}}(y)+\frac{1}{2} j_{\mu}^{\mathrm{e}}(x) j_{\mu}^{\mathrm{e}}(y)\right]+\right. \\
\left.+32(\pi \eta)^{4} \int d^{4} x d^{4} y d^{4} z d^{4} u D_{m}^{(4)}(x-z) D_{m}^{(4)}(y-u) \Sigma_{\mu \nu}^{\mathrm{e}}(x) \Sigma_{\lambda \rho}^{\mathrm{e}}(y)\left\langle\left\langle\Sigma_{\mu \nu}(z) \Sigma_{\lambda \rho}(u)\right\rangle\right\rangle_{x_{\mu}(\xi)}\right\} .
\end{gathered}
$$

Comparing this expression with Eq. (16), we see that owing to Eq. (52), the additional contribution of vortex loops to the cumulant (17) has the form

$$
\begin{gathered}
\Delta\left\langle\left\langle f_{\mu \nu}(x) f_{\lambda \rho}(y)\right\rangle\right\rangle_{a_{\mu}, j_{\mu}^{\mathrm{m}}}=\left(4 \pi g_{m} \eta^{2}\right)^{2} \int d^{4} z \int d^{4} u D_{m}^{(4)}(x-z) D_{m}^{(4)}(y-u) \times \\
\times\left\{\left(\delta_{\mu \lambda} \delta_{\nu \rho}-\delta_{\mu \rho} \delta_{\nu \lambda}\right) \mathcal{G}(z-u)+\left[\delta_{\mu \rho} \partial_{\lambda}^{z} \partial_{\nu}^{z}+\delta_{\nu \lambda} \partial_{\mu}^{z} \partial_{\rho}^{z}-\delta_{\mu \lambda} \partial_{\rho}^{z} \partial_{\nu}^{z}-\delta_{\nu \rho} \partial_{\mu}^{z} \partial_{\lambda}^{z}\right] g(z-u)\right\} .
\end{gathered}
$$

\footnotetext{
${ }^{5}$ Clearly, this result can also be obtained directly by making use of the method presented in the Appendix, which was done in Ref. 26].
} 
Let us further compare this intermediate result with Eq. (17) and take into account that

$$
(x-y)_{\mu} \mathcal{D}_{1}\left((x-y)^{2}\right)=-\frac{1}{2} \partial_{\mu}^{x} G\left((x-y)^{2}\right),
$$

where the function $G$ is defined by Eq. (19). This leads to the following system of equations, which determine the contributions of vortex loops to the functions $\mathcal{D}$ and $G$ :

$$
\begin{aligned}
& \Delta \mathcal{D}\left((x-y)^{2}\right)=\left(4 \pi g_{m} \eta^{2}\right)^{2} \int d^{4} z \int d^{4} u D_{m}^{(4)}(x-z) D_{m}^{(4)}(y-u) \mathcal{G}(z-u), \\
& \Delta G\left((x-y)^{2}\right)=\left(8 \pi g_{m} \eta^{2}\right)^{2} \int d^{4} z \int d^{4} u D_{m}^{(4)}(x-z) D_{m}^{(4)}(y-u) g(z-u) .
\end{aligned}
$$

Inserting now Eq. (47) into Eq. (53), we get

$$
\Delta \mathcal{D}\left((x-y)^{2}\right)=-\frac{\left(4 g_{m} \eta^{2}\right)^{2} \zeta}{\Lambda^{4}} \int d^{4} u D_{m}^{(4)}(y-u) D_{M}^{(4)}(x-u) .
$$

By virtue of the Appendix, this yields

$$
\Delta \mathcal{D}\left(x^{2}\right)=\frac{m^{2}}{4 \pi^{2}}\left[\frac{M}{|x|} K_{1}(M|x|)-\frac{m}{|x|} K_{1}(m|x|)\right] .
$$

Adding this result to Eq. (22), we finally obtain for the finction $\mathcal{D}$ the following full result:

$$
\mathcal{D}^{\text {full }}\left(x^{2}\right)=\frac{m^{2} M}{4 \pi^{2}} \frac{K_{1}(M|x|)}{|x|} .
$$

Analogously, inserting Eq. (51) into Eq. (54), we have

$$
\Delta G\left((x-y)^{2}\right)=\zeta\left(\frac{8 g_{m} \eta^{2}}{\Lambda^{2} M}\right)^{2} \int d^{4} u D_{m}^{(4)}(y-u)\left[D_{0}^{(4)}(x-u)-D_{M}^{(4)}(x-u)\right]
$$

or further by virtue of the Appendix,

$$
\Delta G\left(x^{2}\right)=\left(\frac{m_{D}}{\pi M|x|}\right)^{2}+\left(\frac{2 m}{M}\right)^{2} D_{M}^{(4)}(x)-4 D_{m}^{(4)}(x) .
$$

Together with Eq. (23), this yields the following full result for the function $\mathcal{D}_{1}$ :

$$
\mathcal{D}_{1}^{\text {full }}\left(x^{2}\right)=\frac{m_{D}^{2}}{\pi^{2} M^{2}|x|^{4}}+\frac{m^{2}}{2 \pi^{2} M x^{2}}\left[\frac{K_{1}(M|x|)}{|x|}+\frac{M}{2}\left(K_{0}(M|x|)+K_{2}(M|x|)\right)\right] .
$$

It is worth noting that the functions $\Delta \mathcal{D}$ and $\Delta \mathcal{D}_{1}$ contain the terms exactly equal to Eqs. (22) and (23), respectively, but with the opposite sign, which just cancel out in the full functions (55) and (56). We also see that, as it should be, the functions (55) and (56) go over into Eqs. (22) and (23), respectively, when $m_{D} \rightarrow 0$, i.e. when one neglects the effect of screening in the ensemble of vortex loops. An obvious important consequence of the obtained Eqs. (55) and (56) is that the correlation length of the vacuum, $T_{g}$, becomes modified from $\frac{1}{m}$ (according to Eqs. (22) and (23)) to $\frac{1}{M}$. (It is worth emphasizing once more that this effect is just due to the Debye screening of magnetic charge of the dual vector boson in the ensemble of electrically charged vortex loops, which makes this particle more heavy, namely enlarges its mass from $m$ to $M$.) Indeed, it is straightforward to see that at $|x| \gg \frac{1}{M}$, 


$$
\mathcal{D}^{\text {full }} \longrightarrow \frac{(m M)^{2}}{4 \sqrt{2} \pi^{\frac{3}{2}}} \frac{\mathrm{e}^{-M|x|}}{(M|x|)^{\frac{3}{2}}}
$$

and

$$
\mathcal{D}_{1}^{\text {full }} \longrightarrow \frac{m_{D}^{2}}{\pi^{2} M^{2}|x|^{4}}+\frac{(m M)^{2}}{2 \sqrt{2} \pi^{\frac{3}{2}}} \frac{\mathrm{e}^{-M|x|}}{(M|x|)^{\frac{5}{2}}} .
$$

It is also remarkable that the leading term of the IR asymptotics of the function $\mathcal{D}_{1}^{\text {full }}$ is a pure power-like one, rather than that of the function $\mathcal{D}_{1}$, given by Eq. (25). Another nontrivial result is that the screening does not change the UV asymptotic behaviours of the functions (22) and (23), i.e. the UV asymptotics of the functions (55) and (56) are given by Eqs. (26) and (27), respectively.

Finally, it is worth remarking that due to the modification of the $\mathcal{D}$-function, one could expect the appearance of some change in the string tension of the open dual string world sheet $\Sigma^{\mathrm{e}}$. However, by virtue of the general formula expressing the string tension via the $\mathcal{D}$-function [28], $\sigma=4 T_{g}^{2} \int d^{2} z \mathcal{D}\left(z^{2}\right)$, one can check that this is not the case, i.e. the string tension of $\Sigma^{\mathrm{e}}$ is independent of whether we account for screening in the gas of vortex loops or not. The reason for that becomes clear from the resulting expression for $\sigma$. It reads $16 \pi \eta^{2} \ln \frac{1}{c}$ with $c$ standing for a characteristic small dimensionless quantity, and thus depends only on $\eta$, which is not affected by screening. Similarly to Eq. (37), setting for $c$ the value $\frac{g_{m}}{\sqrt{\lambda}}$, we see that the string tension of $\Sigma^{\mathrm{e}}$ is in the factor 16 larger than the string tension of a vortex loop. Clearly, that is due to the factor 4 standing in the linear combination of $\Sigma_{\mu \nu}$ and $\Sigma_{\mu \nu}^{\mathrm{e}}$ in $\hat{\Sigma}_{\mu \nu}$ (cf. Eq. (15)). However, the coupling constant of the next-to-leading term in the gradient expansion of the nonlocal string effective action standing in the second exponential factor on the R.H.S. of Eq. (15) (the so-called rigidity term) does depend explicitly on the magnetic coupling constant and therefore changes due to the screening. Indeed, by virtue of the results of Refs. [28, 113], one can see that for the same world sheet $\Sigma^{\mathrm{e}}$, this coupling constant without taking screening into account reads $\frac{2 \pi}{\left(2 g_{m}\right)^{2}}$, whereas in the presence of screening it goes over to $\frac{2 \pi}{Q^{2}}$, as it could be intuitively expected.

\section{2 $S U(3)$-case}

In the present Subsection, we shall extend the above results concerning the electric field strength correlators in the gas of vortex loops to the case of AP $S U(3)$-gluodynamics (28). When the dual Nielsen-Olesen strings in this theory are considered as noninteracting objects, the string representation of its partition function is given by Eq. (33), where one should set $\Sigma_{\mu \nu}^{\mathrm{e}}=0$. Integrating out the coordinates of one of the three world sheets (for concreteness, $x_{\mu}^{3}(\xi)$ ), we can write the so-obtained expression for the partition function as

$$
\begin{gathered}
\mathcal{Z}=\int \mathcal{D} x_{\mu}^{1}(\xi) \mathcal{D} x_{\mu}^{2}(\xi) \times \\
\times \exp \left\{-2(\pi \eta)^{2} \int d^{4} x \int d^{4} y\left[\Sigma_{\mu \nu}^{1}(x) \Sigma_{\mu \nu}^{1}(y)+\Sigma_{\mu \nu}^{1}(x) \Sigma_{\mu \nu}^{2}(y)+\Sigma_{\mu \nu}^{2}(x) \Sigma_{\mu \nu}^{2}(y)\right] D_{m_{B}}^{(4)}(x-y)\right\} .
\end{gathered}
$$

In order to proceed from the individual strings to the grand canonical ensemble of vortex loops, one should replace $\Sigma_{\mu \nu}^{a}(x), a=1,2$, in Eq. (57) by 


$$
\Sigma_{\mu \nu}^{a \operatorname{gas}}(x)=\sum_{i=1}^{N} n_{i}^{a} \int d \sigma_{\mu \nu}\left(x_{i}^{a}(\xi)\right) \delta\left(x-x_{i}^{a}(\xi)\right) .
$$

Here, $n_{i}^{a}$ 's stand for winding numbers, which we shall again set to be equal \pm 1 . Performing such a replacement, one can see the crucial difference of the grand canonical ensemble of vortex loops in the model under study from that in the AP $S U(2)$-gluodynamics, studied in the previous Subsection. Namely, the system has now the form of two interacting gases consisting of the vortex loops of two kinds, whereas in the $S U(2)$-case the gas was built out of vortex loops of the only one kind.

Analogously to that case, we shall treat such a grand canonical ensemble of vortex loops in the dilute gas approximation. According to it, characteristic sizes of loops are much smaller than characteristic distances between them, which in particular means that the vortex loops are short living (virtual) objects. Then the summation over this grand canonical ensemble can be most easily performed by inserting the unity

$$
1=\int \mathcal{D} S_{\mu \nu}^{a} \delta\left(S_{\mu \nu}^{a}-\Sigma_{\mu \nu}^{a \text { gas }}\right)
$$

into the R.H.S. of Eq. (57) (where $\Sigma_{\mu \nu}^{a}$ is replaced by $\Sigma_{\mu \nu}^{a \text { gas }}$ ) and representing the $\delta$-function as an integral over the Lagrange multiplier (cf. Eqs. (43) and (45)). Then, the contribution of $N$ vortex loops of each kind to the grand canonical ensemble takes the following form:

$$
\begin{gathered}
\mathcal{Z}\left[\Sigma_{\mu \nu}^{a \text { gas }}\right]=\int \mathcal{D} S_{\mu \nu}^{a} \mathcal{D} \lambda_{\mu \nu}^{a} \times \\
\times \exp \left\{-2(\pi \eta)^{2} \int d^{4} x \int d^{4} y\left[S_{\mu \nu}^{1}(x) S_{\mu \nu}^{1}(y)+S_{\mu \nu}^{1}(x) S_{\mu \nu}^{2}(y)+S_{\mu \nu}^{2}(x) S_{\mu \nu}^{2}(y)\right] D_{m_{B}}^{(4)}(x-y)-\right. \\
\left.-i \int d^{4} x \lambda_{\mu \nu}^{a}\left(S_{\mu \nu}^{a}-\Sigma_{\mu \nu}^{a \text { gas }}\right)\right\} .
\end{gathered}
$$

After that, the desired summation over the ensemble of loops is straightforward, since it technically parallels the one of AP $S U(2)$-gluodynamics. We have

$$
\begin{aligned}
& \prod_{a=1}^{2}\left[1+\sum_{N=1}^{\infty} \frac{\zeta^{N}}{N !}\left\langle\exp \left(i \int d^{4} x \lambda_{\mu \nu}^{a} \Sigma_{\mu \nu}^{a \text { gas }}\right)\right\rangle_{\left\{x_{i}^{a}(\xi)\right\}_{i=1}^{N}}\right]= \\
& =\exp \left\{2 \zeta \int d^{4} y\left[\cos \left(\frac{\left|\lambda_{\mu \nu}^{1}(y)\right|}{\Lambda^{2}}\right)+\cos \left(\frac{\left|\lambda_{\mu \nu}^{2}(y)\right|}{\Lambda^{2}}\right)\right]\right\},
\end{aligned}
$$

where for every $a$, the average $\langle\ldots\rangle_{\left\{x_{i}^{a}(\xi)\right\}_{i=1}^{N}}$ is given by Eq. (44). Here, it has been naturally assumed that the vortex loops of different kinds have the same fugacity $\zeta \propto \mathrm{e}^{-S_{0}}$, since different $\theta_{a}^{\text {sing.' }}$ enter the initial partition function (28) in the symmetric way. Clearly, the action $S_{0}$ of a single loop can be estimated analogously to how it has been done in the previous Subsection for the $S U(2)$-case. In Eq. (60), we have also introduced a new UV momentum cutoff $\Lambda \equiv \sqrt{\frac{L}{a^{3}}}$ $\left(\gg a^{-1}\right)$, where $a$ again denotes a typical size of the vortex loop, whereas $L$ stands for a typical distance between loops, so that in the dilute gas approximation under study $a \ll L$. Finally in Eq. (60), we have denoted $\left|\lambda_{\mu \nu}^{a}\right| \equiv \sqrt{\left(\lambda_{\mu \nu}^{a}\right)^{2}}$. 
Next, it is possible to integrate out the Lagrange multipliers by solving the saddle-point equation following from Eqs. (59) and (60),

$$
\frac{\lambda_{\mu \nu}^{a}}{\left|\lambda_{\mu \nu}^{a}\right|} \sin \left(\frac{\left|\lambda_{\mu \nu}^{a}\right|}{\Lambda^{2}}\right)=-\frac{i \Lambda^{2}}{2 \zeta} S_{\mu \nu}^{a} .
$$

After that, we arrive at the following representation for the partition function of the grand canonical ensemble of vortex loops:

$$
\begin{gathered}
\mathcal{Z}=\int \mathcal{D} S_{\mu \nu}^{a} \exp \left\{-\left[2(\pi \eta)^{2} \int d^{4} x \int d^{4} y\left[S_{\mu \nu}^{1}(x) S_{\mu \nu}^{1}(y)+S_{\mu \nu}^{1}(x) S_{\mu \nu}^{2}(y)+S_{\mu \nu}^{2}(x) S_{\mu \nu}^{2}(y)\right] \times\right.\right. \\
\left.\left.\times D_{m_{B}}^{(4)}(x-y)+V\left[\frac{S_{\mu \nu}^{1}}{\pi}\right]+V\left[\frac{S_{\mu \nu}^{2}}{\pi}\right]\right]\right\},
\end{gathered}
$$

which owing to Eq. (58) is natural to be referred to as the representation in terms of the vortex loops. In Eq. (61), the effective potential of vortex loops is given by Eq. (41).

Next, to get the Debye masses, corresponding to the two interacting gases of vortex loops, it is necessary to get the respective sine-Gordon theory of the two Kalb-Ramond fields. In order to derive it, let us first diagonalize the quadratic form in square brackets on the R.H.S. of Eq. (59), which can be done upon the introduction of the new integration variables $\mathcal{S}_{\mu \nu}^{1}=\frac{\sqrt{3}}{2}\left(S_{\mu \nu}^{1}+S_{\mu \nu}^{2}\right)$ and $\mathcal{S}_{\mu \nu}^{2}=\frac{1}{2}\left(S_{\mu \nu}^{1}-S_{\mu \nu}^{2}\right)$. After that, Eqs. (59) and (60) yield

$$
\begin{aligned}
\mathcal{Z} & =\int \mathcal{D} \mathcal{S}_{\mu \nu}^{a} \mathcal{D} \lambda_{\mu \nu}^{a} \exp \left\{-2(\pi \eta)^{2} \int d^{4} x \int d^{4} y \mathcal{S}_{\mu \nu}^{a}(x) D_{m_{B}}^{(4)}(x-y) \mathcal{S}_{\mu \nu}^{a}(y)+\right. \\
& \left.+2 \zeta \int d^{4} x\left[\cos \left(\frac{\left|\lambda_{\mu \nu}^{1}(x)\right|}{\Lambda^{2}}\right)+\cos \left(\frac{\left|\lambda_{\mu \nu}^{2}(x)\right|}{\Lambda^{2}}\right)\right]-i \int d^{4} x h_{\mu \nu}^{a} \mathcal{S}_{\mu \nu}^{a}\right\} .
\end{aligned}
$$

Here, we have introduced the two Kalb-Ramond fields as the following linear combinations of the Lagrange multipliers: $h_{\mu \nu}^{1}=\frac{1}{\sqrt{3}}\left(\lambda_{\mu \nu}^{1}+\lambda_{\mu \nu}^{2}\right)$ and $h_{\mu \nu}^{2}=\lambda_{\mu \nu}^{1}-\lambda_{\mu \nu}^{2}$. The partition function of the desired sine-Gordon theory can then be obtained from Eq. (62) by making use of the following equality:

$$
\begin{gathered}
\int \mathcal{D} \mathcal{S}_{\mu \nu}^{a} \exp \{- \\
\left.-\left[2(\pi \eta)^{2} \int d^{4} x \int d^{4} y \mathcal{S}_{\mu \nu}^{a}(x) D_{m_{B}}^{(4)}(x-y) \mathcal{S}_{\mu \nu}^{a}(y)+i \int d^{4} x h_{\mu \nu}^{a} \mathcal{S}_{\mu \nu}^{a}\right]\right\}= \\
=\exp \left\{-\frac{1}{2 \pi^{2}} \int d^{4} x\left[\frac{1}{12 \eta^{2}}\left(H_{\mu \nu \lambda}^{a}\right)^{2}+\frac{3}{2} g_{m}^{2}\left(h_{\mu \nu}^{a}\right)^{2}\right]\right\} .
\end{gathered}
$$

Similarly to the analogous equality (38), the equality (63) can easily be proved by noting that due to the Hodge decomposition theorem and the equation $\partial_{\mu} \mathcal{S}_{\mu \nu}^{a}=0$ (which follows from Eq. (58) and conservation of $\Sigma_{\mu \nu}^{a \text { gas }}$ ), $\partial_{\mu} h_{\mu \nu}^{a}=0$. Substituting further Eq. (63) into Eq. (62) and performing the rescaling $\frac{h_{\mu \nu}^{a}}{\pi \sqrt{2}} \rightarrow h_{\mu \nu}^{a}$, we arrive at the following representation for the partition function of the grand canonical ensemble of vortex loops in terms of the local sine-Gordon theory, equivalent to the nonlocal theory (61): 


$$
\begin{gathered}
\mathcal{Z}=\int \mathcal{D} h_{\mu \nu}^{a} \exp \left\{-\int d^{4} x\left[\frac{1}{12 \eta^{2}}\left(H_{\mu \nu \lambda}^{a}\right)^{2}+\frac{3}{2} g_{m}^{2}\left(h_{\mu \nu}^{a}\right)^{2}-\right.\right. \\
\left.\left.-2 \zeta\left[\cos \left(\frac{\pi}{\Lambda^{2} \sqrt{2}}\left|\sqrt{3} h_{\mu \nu}^{1}+h_{\mu \nu}^{2}\right|\right)+\cos \left(\frac{\pi}{\Lambda^{2} \sqrt{2}}\left|\sqrt{3} h_{\mu \nu}^{1}-h_{\mu \nu}^{2}\right|\right)\right]\right]\right\} .
\end{gathered}
$$

The full masses of the Kalb-Ramond fields can now be read off from Eq. (64) by expanding the cosines up to the quadratic terms. The result reads $M_{a}^{2}=m_{B}^{2}+m_{a}^{2} \equiv Q_{a}^{2} \eta^{2}$, where $m_{1}=\frac{2 \pi \eta}{\Lambda^{2}} \sqrt{3 \zeta}$, $m_{2}=\frac{2 \pi \eta}{\Lambda^{2}} \sqrt{\zeta}$ are the Debye masses, and we have introduced the full magnetic charges $Q_{1}=$ $\sqrt{6 g_{m}^{2}+\frac{12 \pi^{2} \zeta}{\Lambda^{4}}}, Q_{2}=\sqrt{6 g_{m}^{2}+\frac{4 \pi^{2} \zeta}{\Lambda^{4}}}$.

Next, Eq. (61) can be used for the evaluation of correlators of vortex loops, which due to Eq. (58) are nothing else but the correlators of $S_{\mu \nu}^{a}$ 's. Those are again calculable in the approximation of a dilute gas of vortex loops, $\left|S_{\mu \nu}^{a}\right| \ll \frac{\zeta}{\Lambda^{2}}$. Within this approximation, the generating functional for correlators of $S_{\mu \nu}^{a}$ 's reads

$$
\begin{gathered}
\mathcal{Z}\left[J_{\mu \nu}^{a}\right]=\int \mathcal{D} \mathcal{S}_{\mu \nu}^{a} \exp \left\{-\left[2(\pi \eta)^{2} \int d^{4} x \int d^{4} y \mathcal{S}_{\mu \nu}^{a}(x) D_{m_{B}}^{(4)}(x-y) \mathcal{S}_{\mu \nu}^{a}(y)+\right.\right. \\
\left.\left.+\int d^{4} x\left[-4 \zeta+\frac{\Lambda^{4}}{2 \zeta}\left(\frac{1}{3}\left(\mathcal{S}_{\mu \nu}^{1}\right)^{2}+\left(\mathcal{S}_{\mu \nu}^{2}\right)^{2}\right)+\mathcal{S}_{\mu \nu}^{1} \frac{J_{\mu \nu}^{+}}{\sqrt{3}}+\mathcal{S}_{\mu \nu}^{2} J_{\mu \nu}^{-}\right]\right]\right\}
\end{gathered}
$$

where $J_{\mu \nu}^{a}$ is a source of $S_{\mu \nu}^{a}$, and $J_{\mu \nu}^{ \pm} \equiv J_{\mu \nu}^{1} \pm J_{\mu \nu}^{2}$. Apart from an inessential constant factor (which can as usual be referred to the integration measure and eventually drops out during the calculation of the correlation functions), we thus get for the generating functional the following expression:

$$
\mathcal{Z}\left[J_{\mu \nu}^{a}\right]=\exp \left\{-\int d^{4} x \int d^{4} y\left[J_{\mu \nu}^{+}(x) J_{\mu \nu}^{+}(y) \mathcal{G}_{1}(x-y)+J_{\mu \nu}^{-}(x) J_{\mu \nu}^{-}(y) \mathcal{G}_{2}(x-y)\right]\right\}
$$

where $\mathcal{G}_{a}(x) \equiv \frac{\zeta}{2 \Lambda^{4}}\left(\partial^{2}-m_{B}^{2}\right) D_{M_{a}}^{(4)}(x)$. Owing to the conservation of $S_{\mu \nu}^{a}$ 's and the Hodge decomposition theorem, we again have the following representation for $S_{\mu \nu}^{a}: S_{\mu \nu}^{a}=\varepsilon_{\mu \nu \lambda \rho} \partial_{\lambda} \varphi_{\rho}^{a}$. Therefore, due to the same theorem, $J_{\mu \nu}^{a}=\varepsilon_{\mu \nu \lambda \rho} \partial_{\lambda} I_{\rho}^{a}$, which yields

$$
\begin{aligned}
\mathcal{Z}\left[J_{\mu \nu}^{a}\right]=\exp & \left\{-2 \int d^{4} x \int d^{4} y\left[I_{\mu}^{a}(x) I_{\nu}^{a}(y) T_{\mu \nu}(x)\left(\mathcal{G}_{1}(x-y)+\mathcal{G}_{2}(x-y)\right)+\right.\right. \\
+ & \left.\left.2 I_{\mu}^{1}(x) I_{\nu}^{2}(y) T_{\mu \nu}(x)\left(\mathcal{G}_{1}(x-y)-\mathcal{G}_{2}(x-y)\right)\right]\right\} .
\end{aligned}
$$

On the other hand, the coupling $\int d^{4} x J_{\mu \nu}^{a} S_{\mu \nu}^{a}$ can be written as $2 \int d^{4} x I_{\mu}^{a} T_{\mu \nu} \varphi_{\nu}^{a}$. Thus, varying $\mathcal{Z}\left[J_{\mu \nu}^{a}\right]$ twice w.r.t. $I_{\mu}^{a}$ 's and setting then $I_{\mu}^{a}=0$, we arrive at the following system of equations:

$$
\begin{gathered}
T_{\mu \nu}(x) T_{\lambda \rho}(y)\left\langle\varphi_{\nu}^{1}(x) \varphi_{\rho}^{1}(y)\right\rangle=T_{\mu \nu}(x) T_{\lambda \rho}(y)\left\langle\varphi_{\nu}^{2}(x) \varphi_{\rho}^{2}(y)\right\rangle=-T_{\mu \lambda}(x)\left(\mathcal{G}_{1}(x-y)+\mathcal{G}_{2}(x-y)\right), \\
T_{\mu \nu}(x) T_{\lambda \rho}(y)\left\langle\varphi_{\nu}^{1}(x) \varphi_{\rho}^{2}(y)\right\rangle=-T_{\mu \lambda}(x)\left(\mathcal{G}_{1}(x-y)-\mathcal{G}_{2}(x-y)\right) .
\end{gathered}
$$


Adopting for the correlators of $\varphi_{\mu}^{a}$ 's the following Ansätze,

$$
\left\langle\varphi_{\nu}^{1}(x) \varphi_{\rho}^{1}(0)\right\rangle=\left\langle\varphi_{\nu}^{2}(x) \varphi_{\rho}^{2}(0)\right\rangle=\delta_{\nu \rho} f_{+}(x), \quad\left\langle\varphi_{\nu}^{1}(x) \varphi_{\rho}^{2}(0)\right\rangle=\delta_{\nu \rho} f_{-}(x),
$$

we get:

$$
f_{ \pm}(x)=\frac{\zeta}{2 \Lambda^{4}}\left(\partial^{2}-m_{B}^{2}\right)\left[\frac{1}{M_{1}^{2}}\left(D_{M_{1}}^{(4)}(x)-D_{0}^{(4)}(x)\right) \pm \frac{1}{M_{2}^{2}}\left(D_{M_{2}}^{(4)}(x)-D_{0}^{(4)}(x)\right)\right] .
$$

This result makes the choice of notations " $f_{ \pm}(x)$ " quite natural. Finally, the desired correlators of vortex loops read

$$
\begin{gathered}
\left\langle S_{\mu \nu}^{1}(x) S_{\lambda \rho}^{1}(0)\right\rangle=\left\langle S_{\mu \nu}^{2}(x) S_{\lambda \rho}^{2}(0)\right\rangle=\left(\delta_{\nu \lambda} \delta_{\mu \rho}-\delta_{\nu \rho} \delta_{\mu \lambda}\right)\left(\mathcal{G}_{1}(x)+\mathcal{G}_{2}(x)\right)+ \\
+\left(\delta_{\mu \lambda} \partial_{\rho} \partial_{\nu}+\delta_{\nu \rho} \partial_{\mu} \partial_{\lambda}-\delta_{\mu \rho} \partial_{\lambda} \partial_{\nu}-\delta_{\nu \lambda} \partial_{\mu} \partial_{\rho}\right) f_{+}(x) \\
\left\langle S_{\mu \nu}^{1}(x) S_{\lambda \rho}^{2}(0)\right\rangle=\left(\delta_{\nu \lambda} \delta_{\mu \rho}-\delta_{\nu \rho} \delta_{\mu \lambda}\right)\left(\mathcal{G}_{1}(x)-\mathcal{G}_{2}(x)\right)+ \\
+\left(\delta_{\mu \lambda} \partial_{\rho} \partial_{\nu}+\delta_{\nu \rho} \partial_{\mu} \partial_{\lambda}-\delta_{\mu \rho} \partial_{\lambda} \partial_{\nu}-\delta_{\nu \lambda} \partial_{\mu} \partial_{\rho}\right) f_{-}(x)
\end{gathered}
$$

This result can now be applied to the calculation of the contribution to the correlator (31), brought about by the vortex loops. Indeed, applying to the average over vortex loops, standing on the R.H.S. of Eq. (34) the cumulant expansion in the bilocal approximation, we have due to Eq. (30):

$$
\begin{gathered}
\Delta\left\langle\left\langle f_{\mu \nu}^{i}(x) f_{\lambda \rho}^{i}(y)\right\rangle\right\rangle_{\mathbf{a}_{\mu}, \mathbf{j}_{\mu}^{M}}= \\
=-24 \pi^{2} g_{m}^{2} \eta^{4} s_{a}^{(c)} s_{b}^{(c)} \int d^{4} z \int d^{4} u D_{m_{B}}^{(4)}(x-z) D_{m_{B}}^{(4)}(y-u)\left\langle S_{\mu \nu}^{a}(z) S_{\lambda \rho}^{b}(u)\right\rangle .
\end{gathered}
$$

Taking further into account the equalities

$$
\left\langle S_{\mu \nu}^{1}(x) S_{\lambda \rho}^{3}(y)\right\rangle=\left\langle S_{\mu \nu}^{2}(x) S_{\lambda \rho}^{3}(y)\right\rangle=\left\langle S_{\mu \nu}^{1}(x) S_{\lambda \rho}^{2}(y)\right\rangle
$$

and the facts that for every $c,\left(s_{a}^{(c)}\right)^{2}=2, s_{1}^{(c)} s_{2}^{(c)}+s_{1}^{(c)} s_{3}^{(c)}+s_{2}^{(c)} s_{3}^{(c)}=-1$, we can write

$$
s_{a}^{(c)} s_{b}^{(c)}\left\langle S_{\mu \nu}^{a}(z) S_{\lambda \rho}^{b}(u)\right\rangle=2\left(\left\langle S_{\mu \nu}^{1}(z) S_{\lambda \rho}^{1}(u)\right\rangle-\left\langle S_{\mu \nu}^{1}(z) S_{\lambda \rho}^{2}(u)\right\rangle\right) .
$$

This leads to the following system of equations:

$$
\begin{gathered}
\Delta \hat{D}\left((x-y)^{2}\right)=48 \pi^{2} g_{m}^{2} \eta^{4} \int d^{4} z \int d^{4} u D_{m_{B}}^{(4)}(x-z) D_{m_{B}}^{(4)}(y-u) \mathcal{G}_{2}(z-u), \\
\Delta \hat{G}\left((x-y)^{2}\right)=96 \pi^{2} g_{m}^{2} \eta^{4} \int d^{4} z \int d^{4} u D_{m_{B}}^{(4)}(x-z) D_{m_{B}}^{(4)}(y-u)\left(f_{+}(z-u)-f_{-}(z-u)\right),
\end{gathered}
$$

where $\hat{G}$ is given by Eq. (19) with the replacement $\mathcal{D}_{1} \rightarrow \hat{D}_{1}$. Carrying now the integrals out analogously to how it was done in the previous Subsection for the $S U(2)$-case, we get 


$$
\begin{gathered}
\Delta \hat{D}\left(x^{2}\right)=m_{B}^{2}\left(D_{M_{2}}^{(4)}(x)-D_{m_{B}}^{(4)}(x)\right) \\
\Delta \hat{G}\left(x^{2}\right)=4\left[\left(\frac{m_{2}}{M_{2}}\right)^{2} D_{0}^{(4)}(x)-D_{m_{B}}^{(4)}(x)+\left(\frac{m}{M_{2}}\right)^{2} D_{M_{2}}^{(4)}(x)\right] .
\end{gathered}
$$

Together with the old expressions for the functions $\hat{D}$ and $\hat{D}_{1}$ (given by Eqs. (22) and (23), respectively, with $m \rightarrow m_{B}$ ), which did not account for the screening effect in the gas of vortex loops 9 , we finally obtain that $\hat{D}^{\text {full }}$ and $\hat{D}_{1}^{\text {full }}$ are given by Eqs. (55) and (56), respectively, with the replacements $m \rightarrow m_{B}, m_{D} \rightarrow m_{2}$, and $M \rightarrow M_{2}$. Therefore the whole discussion, following after Eq. (56), remains the same modulo these replacements. In particular, when $m_{2}$ vanishes, i.e. one disregards the effect of screening, the old expressions for the functions $\hat{D}$ and $\hat{D}_{1}$ are recovered.

\section{Modifications of the Propagators of the Dual Vector Bosons due to the Screening in the Gas of Vortex Loops}

In the present Section, we shall investigate the influence of screening of the dual vector bosons in the gas of vortex loops to the propagators of these bosons themselves. Let us start with the $S U(2)$-case (12) by studying the Wilson loop of a test magnetic particle, whose charge is in the factor $n$ larger than that of the dual Higgs field. Such a Wilson loop has the form

$$
\langle W(C)\rangle=\left\langle\exp \left(2 i g_{m} n \int d^{4} x B_{\mu} j_{\mu}\right)\right\rangle \simeq \exp \left[-2 g_{m}^{2} n^{2} \int d^{4} x \int d^{4} y j_{\mu}(x)\left\langle B_{\mu}(x) B_{\nu}(y)\right\rangle j_{\nu}(y)\right],
$$

where $j_{\mu}(x)=\oint_{C} d x_{\mu}(s) \delta(x-x(s))$, and the average is defined by the partition function (12) with $F_{\mu \nu}^{\mathrm{e}}=0$. Clearly, in the derivation of the last equality on the R.H.S. of Eq. (67) we have used the cumulant expansion in the bilocal approximation. On the other hand, one can derive the string representation for this Wilson loop, and the result reads [24]:

$$
\begin{gathered}
\langle W(C)\rangle=\exp \left[-2 g_{m}^{2} n^{2} \int d^{4} x \int d^{4} y j_{\mu}(x) D_{m}^{(4)}(x-y) j_{\mu}(y)\right] \times \\
\times \frac{1}{\mathcal{Z}} \int \mathcal{D} x_{\mu}(\xi) \exp \left[-(\pi \eta)^{2} \int d^{4} x \int d^{4} y \Sigma_{\mu \nu}(x) D_{m}^{(4)}(x-y) \Sigma_{\mu \nu}(y)+i \int d^{4} x J_{\mu \nu} \Sigma_{\mu \nu}\right],
\end{gathered}
$$

where

$$
J_{\mu \nu}(x) \equiv \pi n \varepsilon_{\mu \nu \lambda \rho} \int d^{4} y j_{\lambda}(y) \partial_{\rho}^{x}\left[D_{m}^{(4)}(x-y)-D_{0}^{(4)}(x-y)\right] .
$$

Using again the bilocal approximation for the average over vortex loops, we have

$$
\langle W(C)\rangle=\exp \left[-2 g_{m}^{2} n^{2} \int d^{4} x \int d^{4} y j_{\mu}(x) D_{m}^{(4)}(x-y) j_{\mu}(y)-\right.
$$

\footnotetext{
${ }^{6}$ It is remarkable that these expressions again become exactly cancelled by the corresponding terms in $\Delta \hat{D}$ and $\Delta \hat{D}_{1}$.
} 


$$
\left.-\frac{1}{2} \int d^{4} x \int d^{4} y J_{\mu \nu}(x)\left\langle S_{\mu \nu}(x) S_{\lambda \rho}(y)\right\rangle J_{\lambda \rho}(y)\right],
$$

where the correlation function of the vortex loops, $\left\langle S_{\mu \nu}(x) S_{\lambda \rho}(y)\right\rangle$, is given by Eq. (52). Inserting now Eqs. (52) and (68) into Eq. (69), comparing the latter one with Eq. (67), using the conservation of $j_{\mu}$ and the fact that $\partial^{2} g=\mathcal{G}$, we get after straightforward calculations:

$$
\begin{gathered}
\left\langle B_{\mu}(x) B_{\nu}(0)\right\rangle=\delta_{\mu \nu}\left[D_{m}^{(4)}(x)+\right. \\
\left.+\frac{g^{2}}{16} \int d^{4} z \int d^{4} u\left(D_{m}^{(4)}(x-z)-D_{0}^{(4)}(x-z)\right) \mathcal{G}(z-u) \partial^{2}\left(D_{m}^{(4)}(u)-D_{0}^{(4)}(u)\right)\right] .
\end{gathered}
$$

Substituting now here the explicit expression (47) for the function $\mathcal{G}$ and using the Appendix for the calculation of the resulting integrals, we eventually arrive at the following simple expression for the propagator of the dual vector boson:

$$
\left\langle B_{\mu}(x) B_{\nu}(0)\right\rangle=\delta_{\mu \nu} \frac{1}{M^{2}}\left(m_{D}^{2} D_{0}^{(4)}(x)+m^{2} D_{M}^{(4)}(x)\right) .
$$

This equation replaces the classical expression $\delta_{\mu \nu} D_{m}^{(4)}(x)$, one has without taking screening into account. Clearly, as it should be, this old expression is reproduced upon taking the limit $m_{D} \rightarrow 0$ in Eq. (71).

Let us now consider the $S U(3)$-case (28), where the magnetic Wilson loop has the form

$$
\begin{gathered}
\left\langle W\left(C_{1}, C_{2}, C_{3}\right)\right\rangle=\left\langle\exp \left(2 i g_{m} n \int d^{4} x \mathbf{B}_{\mu} \mathbf{j}_{\mu}\right)\right\rangle \simeq \\
\simeq \exp \left[-2 g_{m}^{2} n^{2} \int d^{4} x \int d^{4} y j_{\mu}^{a}(x)\left\langle B_{\mu}^{a}(x) B_{\nu}^{b}(y)\right\rangle j_{\nu}^{b}(y)\right] .
\end{gathered}
$$

Similarly to Eq. (9), we have used here the fact that the monopole charges are distributed over the root lattice. Therefore, we have set $\mathbf{j}_{\mu}=\mathbf{e}_{a} j_{\mu}^{a}$, where $j_{\mu}^{a}(x) \equiv \oint_{C_{a}} d x_{\mu}^{(a)}(s) \delta\left(x-x^{(a)}(s)\right)$ with the contour $C_{a}$ parametrized by the vector $x_{\mu}^{(a)}(s)$. We have also introduced the notation $B_{\mu}^{a} \equiv \mathbf{B}_{\mu} \mathbf{e}_{a}$ and used the cumulant expansion in the bilocal approximation. In what follows, we shall be interested in the expression for the propagator $\left\langle B_{\mu}^{a}(x) B_{\nu}^{b}(0)\right\rangle$. To derive it, let us again consider the string representation for the Wilson loop under study. It turns out to be analogous to the one we had in the $S U(2)$-case and reads:

$$
\begin{gathered}
\left\langle W\left(C_{1}, C_{2}, C_{3}\right)\right\rangle=\exp \left[-3 g_{m}^{2} n^{2} \int d^{4} x \int d^{4} y j_{\mu}^{a}(x) D_{m_{B}}^{(4)}(x-y) j_{\mu}^{a}(y)\right] \times \\
\times \frac{1}{\mathcal{Z}} \int \mathcal{D} x_{\mu}^{a}(\xi) \delta\left(\sum_{a=1}^{3} \Sigma_{\mu \nu}^{a}\right) \exp \left[-(\pi \eta)^{2} \int d^{4} x \int d^{4} y \Sigma_{\mu \nu}^{a}(x) D_{m_{B}}^{(4)}(x-y) \Sigma_{\mu \nu}^{a}(y)+i \int d^{4} x J_{\mu \nu}^{a} \Sigma_{\mu \nu}^{a}\right],
\end{gathered}
$$

where

$$
J_{\mu \nu}^{a}(x) \equiv \pi n \varepsilon_{\mu \nu \lambda \rho} \int d^{4} y j_{\lambda}^{a}(y) \partial_{\rho}^{x}\left[D_{m_{B}}^{(4)}(x-y)-D_{0}^{(4)}(x-y)\right] .
$$

Applying the cumulant expansion, we have in the bilocal approximation: 


$$
\begin{gathered}
\left\langle W\left(C_{1}, C_{2}, C_{3}\right)\right\rangle=\exp \left[-3 g_{m}^{2} n^{2} \int d^{4} x \int d^{4} y j_{\mu}^{a}(x) D_{m_{B}}^{(4)}(x-y) j_{\mu}^{a}(y)-\right. \\
\left.-\frac{1}{2} \int d^{4} x \int d^{4} y J_{\mu \nu}^{a}(x)\left\langle S_{\mu \nu}^{a}(x) S_{\lambda \rho}^{b}(y)\right\rangle J_{\lambda \rho}^{b}(y)\right]
\end{gathered}
$$

where the correlation functions of vortex loops, $\left\langle S_{\mu \nu}^{a}(x) S_{\lambda \rho}^{b}(y)\right\rangle$, are defined by Eqs. (65) and (66). These equations, once being inserted into Eq. (74) together with Eq. (73), yield upon some calculations and comparison of the result with Eq. (72):

$$
\begin{gathered}
\left\langle B_{\mu}^{1}(x) B_{\nu}^{1}(0)\right\rangle=\left\langle B_{\mu}^{2}(x) B_{\nu}^{2}(0)\right\rangle= \\
=\frac{1}{3} \delta_{\mu \nu}\left\{\frac{5}{3} D_{m_{B}}^{(4)}(x)+m_{2}^{2}\left[\left(\frac{m_{B}}{m_{1} M_{1}}\right)^{2} D_{M_{1}}^{(4)}(x)+\left(\frac{m_{B}}{m_{2} M_{2}}\right)^{2} D_{M_{2}}^{(4)}(x)+\frac{M_{1}^{2}+M_{2}^{2}}{M_{1}^{2} M_{2}^{2}} D_{0}^{(4)}(x)\right]\right\} \\
\left\langle B_{\mu}^{1}(x) B_{\nu}^{2}(0)\right\rangle= \\
=\frac{1}{3} \delta_{\mu \nu}\left\{\frac{2}{3} D_{m_{B}}^{(4)}(x)+m_{2}^{2}\left[\left(\frac{m_{B}}{m_{1} M_{1}}\right)^{2} D_{M_{1}}^{(4)}(x)-\left(\frac{m_{B}}{m_{2} M_{2}}\right)^{2} D_{M_{2}}^{(4)}(x)-\frac{2 m_{2}^{2}}{M_{1}^{2} M_{2}^{2}} D_{0}^{(4)}(x)\right]\right\} .
\end{gathered}
$$

These equations represent the desired modifications of the propagators of the dual vector bosons in the model (28) due to the Debye screening of the magnetic charges of these bosons in the gas of electric vortex loops. As one can see, in the limit when this effect is disregarded, i.e. $m_{1}, m_{2} \ll m_{B}$, the diagonal propagators (75) go over to the classical expression $\delta_{\mu \nu} D_{m_{B}}^{(4)}(x)$, whereas the off-diagonal one, (76), vanishes. This means that the (quantum) effect of screening leads in particular to the appearance of the nontrivial correlations between dual vector bosons of different types.

\section{Conclusion}

In the present paper, we have investigated field correlators in the AP $S U(2)$ - and $S U(3)$-theories. These correlators are of the two types. First of them are the correlators of electric field strengths, which are the most important ones. That is because they correspond to the gauge-invariant correlators in the real non-Abelian theories, which play the major rôle in the SVM and owing to that are widely used in the phenomenological applications. In AP theories, such correlators have up to now been evaluated only classically. In the present paper, we have improved on these calculations by evaluating the contributions to the correlators, brought about by the screening of the dual vector bosons in the gas of virtual vortex loops. This effect is essentially quantum as well as such a gas itself. In this way, it has been found that the correlation length of the vacuum in the models under study becomes modified from the inverse mass of the dual vector bosons, those acquire by virtue of the Higgs mechanism, to their inverse full mass, which takes also into account the effect of Debye screening. Besides that, in one of the two coefficient functions, which parametrize the bilocal correlator of electric field strengths within the SVM, there appears also a nontrivial power-like IR part, which was absent on the classical level. It has also been checked 
that in the limit when the effect of screening is disregarded, the obtained novel expressions for the bilocal correlators in the AP theories go over to the classical ones, as it should be. It has also been discussed that the found modifications of the bilocal correlators do not affect the string tension, since this quantity depends only on the v.e.v. of the dual Higgs field and not on the mass of the dual vector bosons. Contrary to that, the coupling constant of the so-called rigidity term changes due to the screening.

The correlators of the second kind, we have discussed in this paper, were the propagators of the dual vector bosons themselves. It turned out that (contrary to what happened to the correlation length of the vacuum in electric correlators) in the magnetic propagators the effect of screening does not lead simply to the change of the mass from the pure Higgs to the full one. There rather appear the expressions quite of a novel form, which, however, also go over to the classical ones when the effect of screening is disregarded. Besides that it has been found that in the $S U(3)$-case, screening leads also to the appearance of the nonvanishing correlations between the fields of the dual vector bosons of different kinds. This effect is a purely quantum one and disappears in the classical limit, when the screening is disregarded.

In conclusion, the obtained results shed some light to the vacuum structure of the AP theories and give a new field-theoretical status to the SVM of QCD.

\section{Acknowledgments}

The author is indebted to Prof. A. Di Giacomo for useful discussions and cordial hospitality. He has also benefitted from valuable discussions with Dr. N. Brambilla and Profs. H.G. Dosch and M.G. Schmidt. Besides that, the author is greatful to the whole staff of the Quantum Field Theory Division of the University of Pisa for kind hospitality and INFN for financial support.

\section{Appendix. Calculation of the Integral (49)}

In this Appendix, we shall present some details of calculation of the integral (49). Firstly, owing to the definition of the functions $D_{m}^{(4)}$ and $D_{M}^{(4)}$, we have:

$$
\begin{gathered}
\int d^{4} z D_{m}^{(4)}(z) D_{M}^{(4)}(z-x)=\int \frac{d^{4} p}{(2 \pi)^{4}} \int \frac{d^{4} q}{(2 \pi)^{4}} \int d^{4} z \frac{\mathrm{e}^{i p z}}{p^{2}+m^{2}} \frac{\mathrm{e}^{i q(z-x)}}{q^{2}+M^{2}}= \\
=\int \frac{d^{4} p}{(2 \pi)^{4}} \frac{\mathrm{e}^{i p x}}{\left(p^{2}+m^{2}\right)\left(p^{2}+M^{2}\right)} .
\end{gathered}
$$

Next, this expression can be rewritten as

$$
\int \frac{d^{4} p}{(2 \pi)^{4}} \int_{0}^{+\infty} d \alpha \int_{0}^{+\infty} d \beta \mathrm{e}^{i p x-\alpha\left(p^{2}+m^{2}\right)-\beta\left(p^{2}+M^{2}\right)}=\frac{1}{(4 \pi)^{2}} \int_{0}^{+\infty} d \alpha \int_{0}^{+\infty} d \beta \frac{\mathrm{e}^{-\alpha m^{2}-\beta M^{2}-\frac{x^{2}}{4(\alpha+\beta)}}}{(\alpha+\beta)^{2}} .
$$

It is further convenient to introduce new integration variables $a \in[0,+\infty)$ and $t \in[0,1]$ according to the formulae $\alpha=a t$ and $\beta=a(1-t)$. Then, the integration over $t$ yields for Eq. (A.1) the following expression: 


$$
\frac{1}{\left(4 \pi m_{D}\right)^{2}} \int_{0}^{+\infty} \frac{d a}{a^{2}} \mathrm{e}^{-\frac{x^{2}}{4 a}}\left(\mathrm{e}^{-a m^{2}}-\mathrm{e}^{-a M^{2}}\right) .
$$

Such an integral can be carried out by virtue of the formula

$$
\int_{0}^{+\infty} x^{\nu-1} \mathrm{e}^{-\frac{\beta}{x}-\gamma x} d x=2\left(\frac{\beta}{\gamma}\right)^{\frac{\nu}{2}} K_{\nu}(2 \sqrt{\beta \gamma}), \Re \beta>0, \Re \gamma>0,
$$

and the result has the form of Eq. (50) from the main text. 


\section{References}

[1] H.G. Dosch, Phys. Lett. B 190 (1987) 177; Yu.A. Simonov, Nucl. Phys. B 307 (1988) 512; H.G. Dosch and Yu.A. Simonov, Phys. Lett. B 205 (1988) 339.

[2] H.G. Dosch, Prog. Part. Nucl. Phys. 33 (1994) 121; Yu.A. Simonov, Phys. Usp. 39 (1996) 313.

[3] M. Campostrini, A. Di Giacomo, and G. Mussardo, Z. Phys. C 25 (1984) 173; A. Di Giacomo and H. Panagopoulos, Phys. Lett. B 285 (1992) 133.

[4] L. Del Debbio, A. Di Giacomo, and Yu.A. Simonov, Phys. Lett. B 332 (1994) 111; M. D'Elia, A. Di Giacomo, and E. Meggiolaro, Phys. Lett. B 408 (1997) 315; A. Di Giacomo, E. Meggiolaro, and H. Panagopoulos, Nucl. Phys. B 483 (1997) 371; A. Di Giacomo, M. D'Elia, H. Panagopoulos, and E. Meggiolaro, preprint hep-lat/9808056 (1998); G.S. Bali, N. Brambilla, and A. Vairo, Phys. Lett. B 421 (1998) 265.

[5] O. Nachtmann and A. Reiter, Z. Phys. C 24 (1984) 283; P.V. Landshoff and O. Nachtmann, Z. Phys. C 35 (1987) 405; A. Krämer and H.G. Dosch, Phys. Lett. B 252 (1990) 669; H.G. Dosch, E. Ferreira, and A. Krämer, Phys. Rev. D 50 (1994) 1992.

[6] D.V. Antonov and Yu.A. Simonov, Int. J. Mod. Phys. A 11 (1996) 4401; D.V. Antonov, Int. J. Mod. Phys. A 12 (1997) 2047; Phys. Atom. Nucl. 60 (1997) 299, 478; Yu.A. Simonov, Phys. Atom. Nucl. 61 (1998) 855.

[7] S. Mandelstam, Phys. Rep. C 23 (1976) 245; G. 't Hooft, in: High Energy Physics, Vol. 2, Ed. A. Zichichi (Editrice Compositori, Bologna, 1976), p. 1225; Nucl. Phys. B 190 (1981) 455 .

[8] S. Maedan and T. Suzuki, Prog. Theor. Phys. 81 (1989) 229.

[9] A.M. Polyakov, Nucl. Phys. B 120 (1977) 429.

[10] D.V. Antonov, Mod. Phys. Lett. A 13 (1998) 659; M. Baker, N. Brambilla, H.G. Dosch, and A. Vairo, Phys. Rev. D 58 (1998) 034010; U. Ellwanger, Eur. Phys. J. C 7 (1999) 673. D. Antonov and D. Ebert, Eur. Phys. J. C 8 (1999) 343.

[11] D. Antonov and D. Ebert, Phys. Lett. B 444 (1998) 208; in: Path Integrals from peV to TeV: 50 years after Feynman's paper, Eds. R. Casalbuoni et al. (World Scientific, Singapore, 1999), p. 267; preprint CERN-TH/99-294, hep-th/9909156 (1999) (Nucl. Phys. B (Proc. Suppl.), in press); for related investigations see: D.A. Komarov and M.N. Chernodub, JETP Lett. 68 (1998) 117.

[12] D.V. Antonov, Mod. Phys. Lett. A 13 (1998) 581; D. Antonov and D. Ebert, Eur. Phys. J. C 12 (2000) 349.

[13] D. Antonov, Surveys High Energ. Phys. 14 (2000) 265.

[14] V.N. Popov, Functional Integrals in Quantum Field Theory and Statistical Physics (Reidel, Dordrecht, 1983). 
[15] A.A. Abrikosov, Sov. Phys. JETP 5 (1957) 1174.

[16] H.B. Nielsen and P. Olesen, Nucl. Phys. B 61 (1973) 45.

[17] D. Antonov, Int. J. Mod. Phys. A 14 (1999) 4347.

[18] D. Antonov, Mod. Phys. Lett. A 14 (1999) 1829.

[19] F.A. Schaposnik, Phys. Rev. D 18 (1978) 1183.

[20] Z.F. Ezawa and A. Iwazaki, Phys. Rev. D 25 (1982) 2681; Phys. Rev. D 26 (1982) 631.

[21] K. Bardakci and S. Samuel, Phys. Rev. D 18 (1978) 2849.

[22] K. Lee, Phys. Rev. D 48 (1993) 2493.

[23] M. Kalb and P. Ramond, Phys. Rev. D 9 (1974) 2237.

[24] E.T. Akhmedov et al., Phys. Rev. D 53 (1996) 2087.

[25] P. Orland, Nucl. Phys. B 428 (1994) 221; M. Sato and S. Yahikozawa, Nucl. Phys. B 436 (1995) 100; M. Kiometzis et al., Fortschr. Phys. 43 (1995) 697; E.T. Akhmedov, JETP Lett. 64 (1996) 82; D. Antonov and D. Ebert, in: Problems of Quantum Field Theory, Eds. B.M. Barbashov et al. (JINR, Dubna, 1999), p. 285.

[26] D. Antonov, Phys. Lett. B 475 (2000) 81.

[27] E.M. Lifshitz and L.P. Pitaevski, Statistical Physics, Vol. 2 (Pergamon, New York, 1987).

[28] D.V. Antonov, D. Ebert, and Yu.A. Simonov, Mod. Phys. Lett. A 11 (1996) 1905. 